\title{
Design and Experimental Verification of Robust Motion Synchronization Control with Integral Action
}

\author{
Chen Peng, ${ }^{1}$ Bo Zhu, ${ }^{1}$ and Keqi Wei ${ }^{2}$ \\ ${ }^{1}$ School of Aeronautics and Astronautics, University of Electronic Science and Technology of China, Chengdu, Sichuan 611731, China \\ ${ }^{2}$ Department of Electrical and Computer Engineering, McMaster University, Hamilton, ON, Canada L8S 4K1 \\ Correspondence should be addressed to Chen Peng; penson@buaa.edu.cn
}

Received 24 June 2016; Accepted 19 September 2016

Academic Editor: Steffi Knorn

Copyright (c) 2016 Chen Peng et al. This is an open access article distributed under the Creative Commons Attribution License, which permits unrestricted use, distribution, and reproduction in any medium, provided the original work is properly cited.

\begin{abstract}
A robust attitude motion synchronization problem is investigated for multiple 3-degrees-of-freedom (3-DOF) helicopters with input disturbances. The communication topology among the helicopters is modeled by a directed graph, and each helicopter can only access the angular position measurements of itself and its neighbors. The desired trajectories are generated online and not accessible to all helicopters. The problem is solved by embedding in each helicopter some finite-time convergent (FTC) estimators and a distributed controller with integral action. The FTC estimators generate the estimates of desired angular acceleration and the derivative of the local neighborhood synchronization errors. The distributed controller stabilizes the tracking errors and attenuates the effects of input disturbances. The conditions under which the tracking error of each helicopter converges asymptotically to zero are identified, and, for the cases with nonzero tracking errors, some inequalities are derived to show the relationship between the ultimate bounds of tracking errors and the design parameters. Simulation and experimental results are presented to demonstrate the performance of the controllers.
\end{abstract}

\section{Introduction}

In the field of multivehicle cooperative control, robust consensus tracking under model uncertainties and exogenous disturbances has received increasing attention in recent years, where the output (or state) of each vehicle is required to robustly track a common, desired trajectory. For instance, switching controllers or sliding-mode controllers were designed in works [1-3] to reject input disturbances; a sliding-mode disturbance observer is combined with a consensus tracking algorithm in recent work [4] to improve the robustness and control accuracy of a multimotor system. Alternative robust control approaches include the ones based on uncertainty and disturbance estimators as in works [57], adaptive control approach [8], and the output-regulation approach [9].

In practice, integral control (IC) is widely used to attenuate disturbances in various (single) vehicle systems. This mainly owes to its structural simplicity and the wellknown performance property that IC can asymptotically reject constant input disturbances. Noting these facts, many researchers begin to study IC-based robust control schemes for multivehicle systems (MVSs) as in [10-12]. In particular, the recent work [10] shows that PI controllers successfully attenuate constant disturbances in the network of multiple single-integrator dynamics or the network of multiple double-integrator dynamics.

Another practical issue encountered in many control systems is the lack of sensors. As a result, state observers are often needed to generate the estimates of some necessary states. State observers can be roughly classified into two types: model-dependent ones and model-independent ones. As two representative model-dependent observers, Luenberger observer and Kalman filter suffer from the limitation that the estimation accuracy cannot be guaranteed when the system model suffers from severe uncertainties. To deal with this problem, many model-independent observers are proposed. For instance, some higher-order sliding modes (HOSM) observers (differentiators) were designed in work $[13,14]$ to ensure finite-time convergence even in the presence of input disturbances. 
The main objective of this paper is to use integral control to improve the robustness of a distributed control algorithm for consensus tracking without velocity measurements. The effectiveness of the approach is proved by showing that (a) the resulting tracking errors are ultimately bounded for any input disturbances satisfying a simple Lipschitz-constant condition, and (b) zero-error asymptotic tracking is achieved for a constant input disturbance. The key technical differences between this paper and work [10] are summarized as follows:

(1) The paper [10] considers the consensus problem without a common reference. In contrast, this paper considers a consensus tracking problem, where a common desired trajectory exists (and is supposed to be accessible only to partial vehicles in the group). In [10], velocity signals were used in the control design for second-order systems. We here assume that neither the velocity of leader nor the velocity of neighboring vehicles is accessible for control design.

(2) Concerning the robustness improvement owing to integral control, the discussion in work [10] is restricted to the rather special case with constant input disturbances. This is not the case in this paper. Actually, we will use the concepts of input-to-state stability to study the general cases where the disturbances are nonconstants and are not completely rejected.

(3) In work [10], the controller performance is verified by numerical simulation. In this paper, both numerical simulation results and experimental results on three 3-DOF helicopters are presented, to demonstrate the performance improvement owing to the use of integral control.

The experimental platform of "three 3-DOF helicopters" used in this paper is shown in Figure 1. The single laboratory 3-DOF helicopter with a so-called active disturbance system (ADS) is the same as that in [5] and is shown in Figure 2. This experimental apparatus was developed by Quanser Consulting Inc. for the purpose of control education and research [15-21].

The rest of this paper is organized as follows. In Section 2, some preliminary knowledge is described and the problem is formulated. The robust distributed consensus tracking controllers are designed in Section 3. Numerical simulation and experimental results are presented in Section 4. Some concluding remarks are drawn in Section 5.

\section{Preliminaries}

2.1. Notation and Graph Theory. For matrix $M, M^{-1}$ denotes its inversion and $\operatorname{rank}(M)$ denotes its rank. $I_{n} \in R^{n \times n}$ refers to the identity matrix. For vector $X \in R^{n \times 1}$ and matrix $M=$ $\left[m_{i j}\right] \in R^{m \times n},\|X\|_{2}=\left(\sum_{i=1}^{n}\left|x_{i}\right|^{2}\right)^{1 / 2},\|X\|_{\infty}=\max _{1 \leq i \leq n}\left|x_{i}\right|$, $\|M\|_{\infty}=\max _{1 \leq i \leq m} \sum_{j=1}^{n}\left|m_{i j}\right|$, and $\|M\|_{2}=\left(\lambda_{\max }\left(M^{T} M\right)\right)^{1 / 2}$, where $\lambda_{\max }(\cdot)=\max _{i}\left|\lambda_{i}\right|$ with $\lambda_{i}$ being eigenvalues. $\mathbf{0}_{n}$ refers to $n$-dimensional column vector with all elements being 0 . $|a|$ denotes absolute value (modulus) of real number $a$.

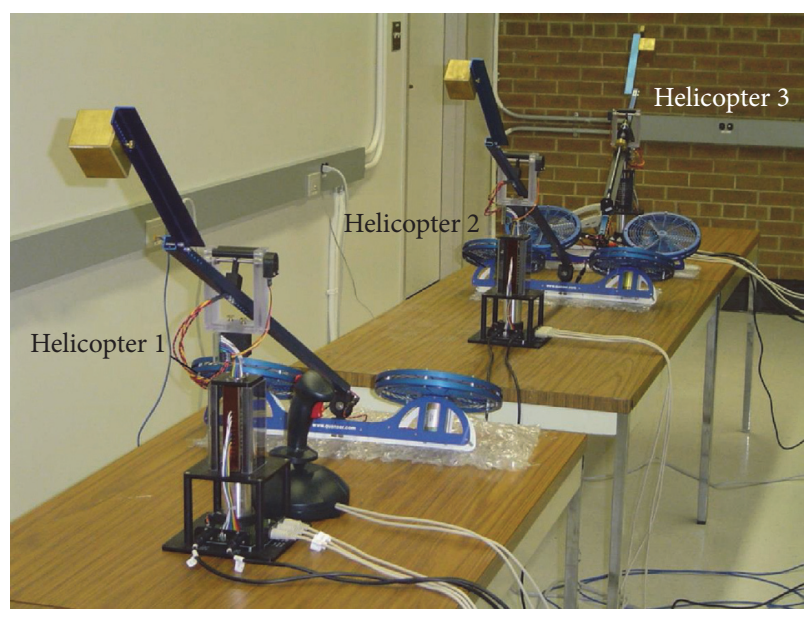

FIGURE 1: Experimental platform of "three 3-DOF helicopters" (see [15]).

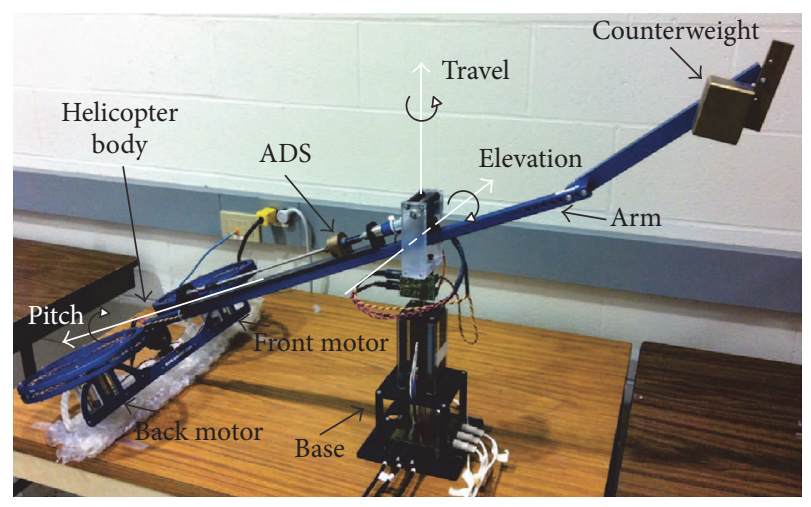

FIGURE 2: 3-DOF helicopter with ADS (see [5]).

The notations related to $i$ th 3 -DOF helicopter (see Figure 2), $i \in \mathscr{I}=\{1, \ldots, n\}$, are listed in Notations.

The communication networks of $n$ helicopters can be modeled by directed graph $\mathscr{G}(\mathscr{V}, \mathscr{E}, \mathscr{A})$, where $\mathscr{V}=$ $\left\{v_{1}, v_{2}, \ldots, v_{n}\right\}, \mathscr{E} \subset \mathscr{V} \times \mathscr{V}$, and nonnegative matrix $\mathscr{A}=$ $\left[a_{i j}\right] \in R^{n \times n}$ denote the set of nodes, the set of edges, and the weighted adjacency matrix of $\mathscr{G}$, respectively. Node $v_{i}$ $(i=1, \ldots, n)$ represents $i$ th helicopter, and an edge $\left(v_{j}, v_{i}\right) \in$ $\mathscr{E}$ denotes that $i$ th helicopter can obtain information from $j$ th helicopter; that is, $v_{j}$ is a neighbor of $v_{i}$. Use $N_{i}$ to denote all the neighbors of $v_{i}$, and $N_{i} \subset \mathscr{V}$. A directed path from $v_{i}$ to $v_{j}$ is a sequence of ordered edges of form $\left(v_{i}, v_{i_{1}}\right),\left(v_{i_{1}}, v_{i_{2}}\right), \ldots,\left(v_{i_{l}}, v_{j}\right)$, with distinct nodes $v_{i_{k}}, k=$ $1,2, \ldots, l$, and $v_{j}$ is said to be reachable from $v_{i}$. A node is called the root node and $\mathscr{G}$ is said to have a directed spanning tree, if all the other nodes are reachable from this node.

The elements of the weighted adjacency matrix $\mathscr{A}$ satisfy $a_{i i}=0$ and $a_{i j}>0(i, j=1, \ldots, n$ and $j \neq i)$ if and only if $\left(v_{j}, v_{i}\right) \in \mathscr{E}$. The Laplacian matrix of $\mathscr{G}$ is denoted by $L=\left[l_{i j}\right] \in R^{n \times n}$, where $l_{i j}=-a_{i j}$, if $i \neq j$, and $l_{i i}=$ $\sum_{j \in N_{i}} a_{i j}$. Note that the desired trajectory information is not accessible to all helicopters in this paper. Use constant matrix 
$\bar{B}=\operatorname{diag}\left(b_{1}, \ldots, b_{n}\right) \in R^{n \times n}$, which is defined as $b_{i}>0$ if $i$ th helicopter can access the desired trajectory information and otherwise $b_{i}=0$, to describe this fact by allowing $b_{i}=0$ for some $i \in \mathscr{I}$.

2.2. Problem Formulation. The elevation and pitch motions of $i$ th helicopter can be modeled as follows (see [5] or [21]):

$$
\begin{aligned}
J_{e i} \ddot{\alpha}_{i}(t)= & K_{f i} l_{a i} \cos \left(\beta_{i}(t)\right) V_{s i}(t)-m_{i} g l_{a i} \cos \left(\alpha_{i}(t)\right) \\
& +f_{\alpha i}(t), \quad i \in \mathscr{I}, \\
J_{p i} \ddot{\beta}_{i}(t)= & K_{f i} l_{h i} V_{d i}(t)+f_{\beta i}(t), \quad i \in \mathscr{I},
\end{aligned}
$$

where

$$
\begin{array}{ll}
V_{s i}(t)=V_{f i}(t)+V_{b i}(t), & i \in \mathscr{F}, \\
V_{d i}(t)=V_{f i}(t)-V_{b i}(t), & i \in \mathscr{I} .
\end{array}
$$

The pitch angle is limited to within $(-\pi / 2, \pi / 2)$ mechanically.

We made the following assumption on $f_{\alpha i}(t)$ and $f_{\beta i}(t)$.

Assumption 1. For each $i \in \mathscr{I}$, the first-order derivatives of $f_{\alpha i}(t)$ and $f_{\beta i}(t)$ with respect to $t$ exist and are piecewise continuous and bounded for all $t \geq 0$.

As shown in recent work [5], by applying the standard normalization and feedback linearization technique, the above nonlinear helicopter model can be reduced to the following 2-dimensional, disturbed double integrators:

$$
\ddot{y}_{i}=u_{i}+d_{i}, \quad i \in \mathscr{I},
$$

where $y_{i}=\left(\alpha_{i}(t), \beta_{i}(t)\right)^{T} \in R^{2}, u_{i}=\left[u_{\alpha i}, u_{\beta i}\right]^{T} \in R^{2}$, and the new disturbances $d_{i}=\left[d_{\alpha i}, d_{\beta i}\right]^{T} \in R^{2}$.

Under Assumption 1, $\dot{d}_{i}=\left(\dot{d}_{\alpha i}, \dot{d}_{\beta i}\right)^{T}$ also exist and are bounded. Define

$$
\begin{aligned}
\bar{d}_{d} & =\left(\bar{d}_{d \alpha}, \bar{d}_{d \beta}\right)^{T} \\
& =\left(\max _{1 \leq i \leq n}\left(\sup _{t \geq 0}\left|\dot{d}_{\alpha i}(t)\right|\right), \max _{1 \leq i \leq n}\left(\sup _{t \geq 0}\left|\dot{d}_{\beta i}(t)\right|\right)\right)^{T},
\end{aligned}
$$

where $\bar{d}_{d \alpha}$ and $\bar{d}_{d \beta}$ are positive scalars.

For each $i \in \mathscr{I}$, we define $y_{i}=\left(\alpha_{i}, \beta_{i}\right)^{T} \in R^{2}$ and use $y_{d}=\left(\alpha_{d}, \beta_{d}\right)^{T} \in R^{2}$ to denote the desired attitude trajectory for $y_{i}$, which may be time-varying but are secondorder differentiable with respect to $t$. Then, the objective of this paper is to design $u_{i}$ for (3) to achieve robust attitude synchronization; that is, $y_{i}(t) \rightarrow y_{d}(t)$ for each $i \in \mathscr{I}$ as $t \rightarrow+\infty$.

\section{Design of Distributed Controllers without Velocity Measurements}

3.1. Controller Design. Noting that $u_{\alpha i}$ and $u_{\beta i}$ for (3) can be designed in the same way, we thus introduce new unified variable $\rho \in\{\alpha, \beta\}$ and define

$$
\begin{aligned}
\tilde{\rho}_{i} & =\rho_{i}-\rho_{d}, \quad i \in \mathscr{I}, \\
\widetilde{\boldsymbol{\rho}} & =\left[\widetilde{\rho}_{1}, \ldots, \widetilde{\rho}_{n}\right]^{T} \in R^{n}, \\
\widetilde{\rho}_{i j} & =\rho_{i}-\rho_{j}, \quad i, j \in \mathscr{I}, \\
e_{\rho i} & =b_{i} \widetilde{\rho}_{i}+\sum_{j \in N_{i}} a_{i j} \widetilde{\rho}_{i j} .
\end{aligned}
$$

In $[8,22]$, error $e_{\rho i}$ is called local neighborhood synchronization error (LNSE).

For the considered helicopter, only angular position sensors (encoders) are equipped. Besides, we assume that the desired velocity is not accessible to any helicopter. To deal with this problem, we consider the following distributed controllers:

$$
\begin{aligned}
& u_{\rho i}(t)=\frac{1}{k_{i}}\left(\sum_{j \in N_{i}} a_{i j} u_{\rho j}(t)+b_{i} \widehat{\ddot{\rho}}_{d}(t)-k_{\rho i}^{P} e_{\rho i}(t)\right. \\
& \left.-k_{\rho i}^{D} \hat{\dot{e}}_{\rho i}(t)-k_{\rho i}^{I} \int_{0}^{t} e_{\rho i}(\tau) d \tau\right),
\end{aligned}
$$

where $k_{\rho i}^{P}, k_{\rho i}^{I}$, and $k_{\rho i}^{D}$ are positive control gains, $k_{i}=b_{i}+$ $\sum_{j \in N_{i}} a_{i j}, \widehat{\ddot{\rho}}_{d}(t)$, and $\hat{\dot{e}}_{\rho i}(t)$ denote the estimates of $\ddot{\rho}_{d}(t)$ and $\dot{e}_{\rho i}(t)$, respectively. We construct the following systems to generate $\widehat{\ddot{\rho}}_{d}(t)$ and $\hat{\dot{e}}_{\rho i}(t)$. Specifically, the third-order FTC estimators

$$
\begin{aligned}
\dot{\hat{\rho}}_{d}= & -\lambda_{2} \bar{L}_{\rho d}^{1 / 3}\left|\hat{\rho}_{d}-\rho_{d}\right|^{2 / 3} \operatorname{sign}\left(\hat{\rho}_{d}-\rho_{d}\right) \\
& -\mu_{2}\left(\hat{\rho}_{d}-\rho_{d}\right)+\hat{\dot{\rho}}_{d}, \\
\dot{\hat{\dot{\rho}}}_{d}= & -\lambda_{1} \bar{L}_{\rho d}^{1 / 2}\left|\hat{\dot{\rho}}_{d}-\dot{\hat{\rho}}_{d}\right|^{1 / 2} \operatorname{sign}\left(\hat{\dot{\rho}}_{d}-\dot{\hat{\rho}}_{d}\right) \\
& -\mu_{1}\left(\hat{\dot{\rho}}_{d}-\dot{\hat{\rho}}_{d}\right)+\widehat{\hat{\rho}}_{d}, \\
\dot{\hat{\dot{\rho}}}_{d}= & -\lambda_{0} \bar{L}_{\rho d} \operatorname{sign}\left(\hat{\ddot{\rho}}_{d}-\dot{\hat{\dot{\rho}}}_{d}\right)-\mu_{0}\left(\widehat{\ddot{\rho}}_{d}-\dot{\hat{\dot{\rho}}}_{d}\right),
\end{aligned}
$$

are for $\widehat{\hat{\rho}}_{d}(t)$, and the following second-order FTC estimators are for $\hat{\dot{e}}(t)$ :

$$
\begin{aligned}
\dot{\hat{e}}_{\rho i}= & -\lambda_{1} \bar{L}_{e}^{1 / 2}\left|\widehat{e}_{\rho i}-e_{\rho i}\right|^{1 / 2} \operatorname{sign}\left(\widehat{e}_{\rho i}-e_{\rho i}\right) \\
& -\mu_{1}\left(\widehat{e}_{\rho i}-e_{\rho i}\right)+\hat{\dot{e}}_{\rho i}, \\
\dot{\dot{e}}_{\rho i}= & -\lambda_{0} \bar{L}_{e} \operatorname{sign}\left(\hat{\dot{e}}_{\rho i}-\dot{\hat{e}}_{\rho i}\right)-\mu_{0}\left(\hat{\dot{e}}_{\rho i}-\dot{\hat{e}}_{\rho i}\right), \quad i \in \mathscr{I},
\end{aligned}
$$

where $\widehat{\rho}_{d}, \hat{\dot{\rho}}_{d}$, and $\widehat{e}_{\rho i}$ are the estimates of $\rho_{d}$, $\dot{\rho}_{d}$, and $e_{\rho i}$, respectively; $\rho_{d}$ and $e_{\rho i}$ are consisting of a locally bounded 


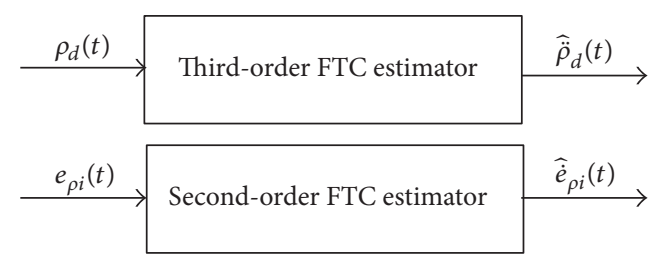

FIGURE 3: The way to obtain $\widehat{\ddot{\rho}}_{d}(t)$ and $\widehat{\dot{e}}(t)$.

Lebesgue-measurable noise with unknown features and an unknown base signal $\rho_{d 0}(t)$ and $e_{\rho i 0}$ whose second and first derivative have an known Lipschitz constant $\bar{L}_{\rho d}>0$ and $\bar{L}_{e}>0$, respectively; $\lambda_{i}$ and $\mu_{i}>0, i=0,1,2$, are the design parameters of the FTC estimators.

This estimation scheme is illustrated in Figure 3.

We have the following result for the above two FTC estimators [14, Theorem 1].

Lemma 2. Let $\left\{\lambda_{i}\right\}$ and $\left\{\mu_{i}\right\}$ in (7) and (8) be recursively chosen as in Theorem 1 of $[13,14]$. Then, the estimation errors achieve zero in the absence of input noises after finite time $t_{c}$ of transient process. The convergence of the estimation errors is uniform in the sense that the convergence time is uniformly bounded by finite time $t_{c}$ which is a locally bounded function of initial estimation errors.

Remark 3. According to Lemma 2, in the absence of input noises, after finite time $t_{c i}(i \in \mathscr{I})$, the following equalities hold:

$$
\begin{aligned}
& \hat{\dot{\rho}}_{d}-\dot{\rho}_{d}=0, \\
& \widehat{\ddot{\rho}}_{d}-\ddot{\rho}_{d}=0, \\
& \dot{e}_{\rho i}-\widehat{\dot{e}}_{\rho i}=0, \quad i \in \mathscr{I} .
\end{aligned}
$$

Remark 4. Note that the neighbors' control signals $u_{\rho j}(t)(j \in$ $N_{i}$ ) are used in (6) and then a possible implementation loop issue arises in practical applications. As discussed in work [5], if the sample frequency is high enough, this problem can be resolved with using the neighbors' control signals obtained during the previous sampling period, that is, $u_{\rho j}(t-\tau)$, where $\tau$ denotes the fixed sampling step and $\tau=0.001 \mathrm{sec}$ in the following numerical simulations and experiments.

3.2. Analysis of the Closed-Loop Stability. Before the closedloop stability analysis, we need to establish and analyse the relationship between tracking errors $\widetilde{\rho}_{i}$ and LNSEs $e_{\rho i}, i \in \mathscr{I}$. Define

$$
\mathbf{E}_{\rho}=\left[e_{\rho 1}, \ldots, e_{\rho n}\right]^{T} \in R^{n}, \quad \rho \in\{\alpha, \beta\} .
$$

From (5c), $\widetilde{\rho}_{i j}=\rho_{i}-\rho_{j}=\widetilde{\rho}_{i}-\widetilde{\rho}_{j}$; then from (5d), $e_{\rho i}=$ $b_{i} \widetilde{\rho}_{i}+\sum_{j \in N_{i}} a_{i j}\left(\widetilde{\rho}_{i}-\widetilde{\rho}_{j}\right)$ for each $\rho \in\{\alpha, \beta\}$. Then, with $L$ and $\bar{B}$ as defined in Section 2.1, the following relationship equation between the tracking errors and LNSEs is derived:

$$
\mathbf{E}_{\rho}(t)=(L+\bar{B}) \tilde{\boldsymbol{\rho}}(t), \quad \rho \in\{\alpha, \beta\},
$$

where $\operatorname{rank}(L+\bar{B})$ is determined by the communication topology which satisfies the following condition in this paper.

Assumption 5. The desired trajectory information $\rho_{d}, \rho \in$ $\{\alpha, \beta\}$, has directed paths to all helicopters.

According to [23, Lemma 1.6], all eigenvalues of $L+\bar{B}$ have positive real parts under Assumption 5. Then, the following lemma is obtained directly.

Lemma 6 (see [23, Lemma 1.6]). Under Assumption 5, matrix $L+\bar{B}$ is full rank, that is, $\operatorname{rank}(L+\bar{B})=n$.

Thus, the relationship equation (11) implies that, under Assumption 5, the objective $\widetilde{\boldsymbol{\rho}}(t) \rightarrow \mathbf{0}_{n}$ and $\dot{\tilde{\boldsymbol{\rho}}}(t) \rightarrow \mathbf{0}_{n}$ as $t \rightarrow+\infty, \rho \in\{\alpha, \beta\}$, can be achieved by driving $\mathbf{E}_{\rho}(t)$ and $\dot{\mathbf{E}}_{\rho}(t)$ to zero as $t \rightarrow+\infty$, respectively.

Applying (6) to (3) gives

$$
\begin{aligned}
k_{i} \ddot{\rho}_{i}(t)= & \sum_{j \in N_{i}} a_{i j}\left(\ddot{\rho}_{j}(t)-d_{\rho j}(t)\right)(t)+b_{i} \widehat{\ddot{\rho}}_{d}(t) \\
& -k_{\rho i}^{P} e_{\rho i}(t)-k_{\rho i}^{D} \hat{e}_{\rho i}(t)-k_{\rho i}^{I} \int_{0}^{t} e_{\rho i}(\tau) d \tau \\
& +k_{i} d_{\rho i} .
\end{aligned}
$$

With (5d) and (12), we can further get

$$
\begin{aligned}
& \ddot{e}_{\rho i}+k_{\rho i}^{D} \ddot{e}_{\rho i}+k_{\rho i}^{P} \dot{e}_{\rho i}+k_{\rho i}^{I} e_{\rho i}=\dot{\delta}_{\rho i}, \\
& \qquad \rho \in\{\alpha, \beta\}, i \in \mathscr{I},
\end{aligned}
$$

where $\delta_{\rho i}=b_{i}\left(\widehat{\ddot{\rho}}_{d}-\ddot{\rho}_{d}\right)+k_{\rho i}^{D}\left(\dot{e}_{\rho i}-\hat{\dot{e}}_{\rho i}\right)+\Delta_{\rho i}$ and $\Delta_{\rho i}$ is defined as

$$
\Delta_{\rho i}=k_{i} d_{\rho i}-\sum_{j \in N_{i}} a_{i j} d_{\rho j}
$$

Define

$$
\begin{aligned}
& \boldsymbol{\delta}_{\rho}=\left[\delta_{\rho 1}, \ldots, \delta_{\rho n}\right]^{T} \in R^{n}, \quad \rho \in\{\alpha, \beta\}, \\
& \Delta_{\rho}=\left[\Delta_{\rho 1}, \ldots, \Delta_{\rho n}\right]^{T} \in R^{n}, \quad \rho \in\{\alpha, \beta\}, \\
& \mathbf{d}_{\rho}=\left[d_{\rho 1}, \ldots, d_{\rho n}\right]^{T} \in R^{n}, \quad \rho \in\{\alpha, \beta\}, \\
& \overline{\mathbf{t}}_{c}=\left[t_{c 1}, \ldots, t_{c n}\right]^{T} \in R^{n} .
\end{aligned}
$$

Then, the following result is clear based on Lemma 2.

Lemma 7. There exists $\bar{t}_{c \max }=\left\|\overline{\mathbf{t}}_{c}\right\|_{\infty}$ such that, in the absence of input noises,

$$
\boldsymbol{\delta}_{\rho}=\Delta_{\rho}, \quad \forall t \geq \bar{t}_{c \max }, \rho \in\{\alpha, \beta\} .
$$

Therefore, after finite time $\bar{t}_{c \max },(13)$ is the same as follows:

$$
\begin{aligned}
& \ddot{e}_{\rho i}+k_{\rho i}^{D} \ddot{e}_{\rho i}+k_{\rho i}^{P} \dot{e}_{\rho i}+k_{\rho i}^{I} e_{\rho i}=\dot{\Delta}_{\rho i}, \\
& \qquad \rho \in\{\alpha, \beta\}, i \in \mathscr{I} .
\end{aligned}
$$


Define

$$
\begin{aligned}
& A_{\rho i}=\left[\begin{array}{ccc}
0 & 1 & 0 \\
0 & 0 & 1 \\
-k_{\rho i}^{I} & -k_{\rho i}^{P} & -k_{\rho i}^{D}
\end{array}\right] \in R^{3 \times 3}, \quad \rho \in \alpha, \beta, i \in \mathscr{I}, \\
& B_{\rho i}=\left[\begin{array}{lll}
0 & 0 & 1
\end{array}\right]^{T} \in R^{3 \times 1}, \quad \rho \in \alpha, \beta, i \in \mathscr{I} .
\end{aligned}
$$

Then, (20) can be written as

$$
\begin{aligned}
{\left[\dot{e}_{\rho i}, \ddot{e}_{\rho i}, \ddot{e}_{\rho i}\right]^{T}=A_{\rho i}\left[e_{\rho i}, \dot{e}_{\rho i}, \ddot{e}_{\rho i}\right]^{T}+} & B_{\rho i} \dot{\Delta}_{\rho i}, \\
& \rho \in \alpha, \beta, i \in \mathscr{I} .
\end{aligned}
$$

Furthermore, based on Lemma 7, by applying Routh-Hurwitz stability criterion and Lemma A.1 in Appendix to forced systems (13) with $\dot{\delta}_{\rho i}$ as inputs, the following theorem is obtained.

Theorem 8. Consider systems (13) under Assumptions 1 and 5 and the following parameter condition:

$$
\begin{aligned}
& k_{\rho i}^{D}, k_{\rho i}^{P}, k_{\rho i}^{I}>0, \\
& k_{\rho i}^{D} k_{\rho i}^{P}>k_{\rho i}^{I} .
\end{aligned}
$$

Then, for each $\rho \in\{\alpha, \beta\}$, in the absence of noise,

(1) the system trajectories $\mathbf{E}_{\rho}, \dot{\mathbf{E}}_{\rho}, \tilde{\boldsymbol{\rho}}$, and $\dot{\tilde{\boldsymbol{\rho}}}$ are globally bounded;

(2) $\mathbf{E}_{\rho} \rightarrow 0, \dot{\mathbf{E}}_{\rho} \rightarrow 0, \widetilde{\boldsymbol{\rho}} \rightarrow 0$, and $\dot{\tilde{\boldsymbol{\rho}}} \rightarrow 0$ as $t \rightarrow+\infty$ if $\dot{\mathbf{d}}_{\rho}(t) \rightarrow 0$ as $t \rightarrow+\infty$;

(3) for the general cases with nonvanishing $\dot{\mathbf{d}}_{\rho}(t)$, there exists $\bar{t}_{\rho}>\bar{t}_{c \text { max }}$ such that, under any initial condition,

$$
\begin{aligned}
\left\|\mathbf{E}_{\rho}(t)\right\|_{2} & \leq b_{\rho}, \quad \forall t \geq \bar{t}_{\rho}, \\
\|\widetilde{\boldsymbol{\rho}}(t)\|_{2} & \leq\left\|(L+\bar{B})^{-1}\right\|_{2} b_{\rho}, \quad \forall t \geq \bar{t}_{\rho},
\end{aligned}
$$

where

$$
\begin{aligned}
b_{\rho}= & \frac{2 \sqrt{n}\|L+\bar{B}\|_{\infty} \bar{d}_{d \rho}}{\theta} \\
& \cdot \max _{1 \leq i \leq n}\left(\lambda_{\max }\left(P_{\rho i}\right) \sqrt{\frac{\lambda_{\max }\left(P_{\rho i}\right)}{\lambda_{\min }\left(P_{\rho i}\right)}}\right),
\end{aligned}
$$

and $0<\theta<1, P_{\rho i}$ are the solutions of the Lyapunov equations

$$
P_{\rho i} A_{\rho i}+A_{\rho i}^{T} P_{\rho i}=-I_{3}, \quad \rho \in\{\alpha, \beta\},
$$

with $A_{\rho i}$ defined by (21).

Proof. Using (14), (16), and (17), the relationships between $\dot{\Delta}_{\rho i}$ and $\dot{d}_{\rho i}$ can be written in a compact form as

$$
\dot{\Delta}_{\rho}=(L+\bar{B}) \dot{\mathbf{d}}_{\rho}, \quad \rho \in\{\alpha, \beta\} .
$$

Then, with (4), it follows that

$$
\begin{aligned}
\left\|\dot{\Delta}_{\rho}(t)\right\|_{\infty} \leq\|L+\bar{B}\|_{\infty}\left\|\dot{\mathbf{d}}_{\rho}(t)\right\|_{\infty} & \leq\|L+\bar{B}\|_{\infty} \bar{d}_{d \rho}, \\
& \forall t \geq 0, \rho \in\{\alpha, \beta\} .
\end{aligned}
$$

Moreover, for $\rho \in\{\alpha, \beta\}$, based on Lemma 6 , under Assumption $5, \dot{\Delta}_{\rho} \rightarrow \mathbf{0}_{n}$ as $t \rightarrow+\infty$ if and only if $\dot{\mathbf{d}}_{\rho} \rightarrow \mathbf{0}_{n}$ as $t \rightarrow+\infty$. Thus, under Assumptions 1 and 5 and parameter conditions (24), it is straightforward to derive the points (1)-

(2) with these results and Lemmas 6 and 7.

The detailed proof of point (3) is the same as [5, Theorem 2] and hence omitted.

Remark 9. As shown in Theorem 8, by FTC differentiators (7) and (8) in the absence of input noises, for the case without angular velocity measurements, if condition $\lim _{t \rightarrow+\infty} \dot{d}_{\rho i}(t)=0$ is satisfied, the distributed controllers (6) are capable of achieving zero-error attitude-trajectory tracking for each helicopter in the group of helicopters. Thus, the distributed consensus attitude-trajectory zero-error tracking for the group of helicopters is achieved under this condition $\left(\lim _{t \rightarrow+\infty} \dot{d}_{\rho i}(t)=0\right)$. If this condition is not satisfied, the ultimate bounds of attitude-trajectory tracking errors $\widetilde{\rho}_{i}$ resulting from controllers (6) are globally bounded for $i \in \mathscr{I}$ and ultimately bounded with ultimate bounds depending on parameters $k_{\rho i}^{P}, k_{\rho i}^{D}, k_{\rho i}^{I}$ which can make the term $\max _{1 \leq i \leq n}\left(\lambda_{\max }\left(P_{\rho i}\right) \sqrt{\lambda_{\max }\left(P_{\rho i}\right) / \lambda_{\text {min }}\left(P_{\rho i}\right)}\right)$ small enough. Future efforts will be devoted to the optimization problem of the three parameters such that the term is minimized.

Remark 10. If delay $\tau$ in neighbors' control inputs $u_{\rho j}(t-\tau)$ is systematically considered, the obtained error equations (13) are disturbed neutral delay systems as in [24]. For these kinds of delayed systems, [25, Lemma 1] can be used to examine the stability of the associated nominal delayed systems, where three easily testable conditions are included. We also note that these conditions ensure that the considered systems are delayindependent stable. However, the delay-independent stability is not the general case. Despite this fact, since the case with $\tau=0$ is exponentially stable, we readily derive that the system with an enough small time-delay is also exponentially stable, which ensure that the correspondingly disturbed equations are input-to-state stable provided that the delay is sufficiently small. In our future work, we will investigate these problems and systematically assess the effect of the introduced delay on the system stability as well as the convergence speed of tracking errors (as in the work [26]).

\section{Numerical Simulation and Experimental Results}

In this section, to demonstrate the effectiveness of the proposed scheme for robust motion synchronization, the numerical simulation results and experimental results of the attitude-trajectory consensus tracking of three 3-DOF helicopters which are labeled as $\mathrm{H} 1$ to $\mathrm{H} 3$ are presented. 
TABLE 1: Nominal parameters of the helicopters $(i=0,1,2,3)$.

\begin{tabular}{lc}
\hline Parameter & Value \\
\hline$K_{f i}$ & $0.1188 \mathrm{~N} / \mathrm{V}$ \\
$l_{a i}$ & $0.660 \mathrm{~m}$ \\
$l_{h i}$ & $0.178 \mathrm{~m}$ \\
$m_{i}$ & $0.094 \mathrm{~kg}$ \\
$J_{e i}$ & $1.034 \mathrm{~kg} \cdot \mathrm{m}^{2}$ \\
$J_{p i}$ & $0.045 \mathrm{~kg} \cdot \mathrm{m}^{2}$ \\
$g$ & $9.81 \mathrm{~m} / \mathrm{s}^{2}$ \\
\hline
\end{tabular}

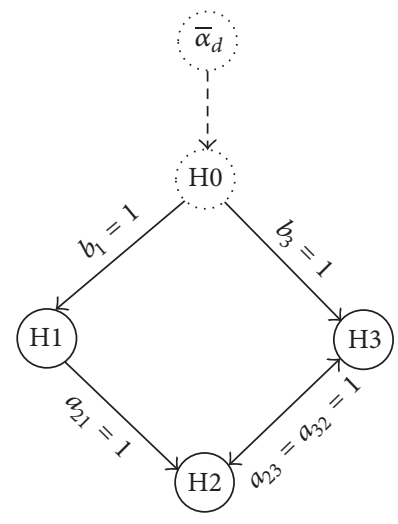

FIGURE 4: The considered communication topology.

Note that we focus on the analysis of the results of elevationtrajectory consensus tracking hereafter. The results of pitch channel are similar to that of elevation channel and hence omitted.

The nominal parameters of the four helicopters (including virtual helicopter $\mathrm{H} 0$ ) are the same, which are presented in Table 1. Their initial states are specified as follows:

$$
\begin{aligned}
& \left(\alpha_{0}(0), \dot{\alpha}_{0}(0)\right)=(-20.0 \mathrm{deg}, 0 \mathrm{deg} / \mathrm{s}), \\
& \left(\alpha_{1}(0), \dot{\alpha}_{1}(0)\right)=(-21.5 \mathrm{deg}, 0 \mathrm{deg} / \mathrm{s}), \\
& \left(\alpha_{2}(0), \dot{\alpha}_{2}(0)\right)=(-27.0 \mathrm{deg}, 0 \mathrm{deg} / \mathrm{s}), \\
& \left(\alpha_{3}(0), \dot{\alpha}_{3}(0)\right)=(-16.9 \mathrm{deg}, 0 \mathrm{deg} / \mathrm{s}) .
\end{aligned}
$$

All the results are obtained on the directed communication graph shown in Figure 4. In this graph, helicopters 1 and 3 have access to desired trajectories $\alpha_{d}(t)$ which are the responses of $\mathrm{H} 0$ to desired signal $\bar{\alpha}_{d}(t)$, and Assumption 5 holds with $b_{1}=b_{3}=a_{21}=a_{23}=a_{32}=1$ and all other entries of $\bar{B}$ and $A_{n}$ being 0 . Then,

$$
L=\left[\begin{array}{ccc}
0 & 0 & 0 \\
-1 & 2 & -1 \\
0 & -1 & 1
\end{array}\right]
$$

TABLE 2: Design parameters of the FTC estimators.

\begin{tabular}{lcc}
\hline Parameter & Equation $(7)$ & Equation $(8)$ \\
\hline$\lambda_{0}$ & 1.1 & 5 \\
$\lambda_{1}$ & 1.5 & 8 \\
$\lambda_{2}$ & 2 & N/A \\
$\mu_{0}$ & 3 & 8 \\
$\mu_{1}$ & 6 & 12 \\
$\mu_{2}$ & 8 & N/A \\
$\bar{L}_{\rho d}$ & 1 & N/A \\
$\bar{L}_{e}$ & N/A & 1 \\
\hline
\end{tabular}

$$
\begin{aligned}
\bar{B} & =\left[\begin{array}{lll}
1 & 0 & 0 \\
0 & 0 & 0 \\
0 & 0 & 1
\end{array}\right], \\
L+\bar{B} & =\left[\begin{array}{ccc}
1 & 0 & 0 \\
-1 & 2 & -1 \\
0 & -1 & 2
\end{array}\right] .
\end{aligned}
$$

Without loss of generality, the following nonstep desired trajectories provided to $\mathrm{H} 0$ are adopted for numerical simulations and experiments:

$$
\begin{aligned}
& \bar{\alpha}_{d}(t)=10 \sin (0.04 \pi t)-5, \\
& \dot{\bar{\alpha}}_{d}(t)=0.4 \pi \cos (0.04 \pi t), \\
& \ddot{\bar{\alpha}}_{d}(t)=-0.016 \pi^{2} \sin (0.04 \pi t),
\end{aligned}
$$

where all the angles are given in degrees. For the parameters of control signal $u_{\alpha 0}$ which is determined by (6) with $b_{0}=1$, $a_{0 j}=0, j \in \mathscr{T}, \widehat{\ddot{\alpha}}_{d}(t)=\ddot{\bar{\alpha}}_{d}(t)$, and $\hat{\dot{e}}_{\alpha 0}(t)=\dot{e}_{\alpha 0}(t)$, choose

$$
\begin{aligned}
& k_{\alpha 0}^{P}=1.5, \\
& k_{\beta 0}^{D}=2, \\
& k_{\alpha 0}^{I}=0,
\end{aligned}
$$

where $\mathrm{H} 0$ is a virtual helicopter without the acting of LUDs; hence the integral term in the control law (6) is not adopted by H0 (i.e., $k_{\alpha 0}^{I}=0$ ) for simplicity.

The design parameters chosen for the FTC estimators (7) and (8) are shown in Table 2. The initial state values of the differentiators in (7) and (8) are set to zero; that is, $\hat{\rho}_{d}(0)=$ $\widehat{\dot{\rho}}_{d}(0)=\widehat{\ddot{\rho}}_{d}(0)=0$ and $\widehat{e}_{\rho i}(0)=\widehat{\dot{e}}_{\rho i}(0)=0, i \in \mathscr{I}$.

In the following, for comparison purposes, six cases (Cases 1-6) are considered. In each case, $u_{\alpha i}$ are determined by (6). In order to theoretically verify the results of Theorem 8, Cases 1-3 are designed for the numerical simulations. Specifically, to demonstrate that both tracking and synchronization performance without IC are not acceptable, IC is not adopted in Case 1; points (1)-(2) of Theorem 8 are verified by Case 2, where the helicopters with IC are subject to different 
constant disturbances; the helicopters with IC are subject to different time-varying disturbances in Case 3 to show the validity of points (1) and (3) of Theorem 8 . The results of Theorem 8 are experimentally verified by Cases $4-6$ on the experiment platform shown in Figure 1. IC is adopted in Cases 5-6 while, in Case 4, it is not. The ADSs of helicopters 2 and 3 are activated in Case 6 while in Cases 4-5 are not. Note that, since the disturbances are unknown in the experiments, the control parameters of $u_{\alpha i}$ in experiments are not the same as those in numerical simulations, where the disturbances are specified and used to choose the control parameters.

The detailed differences among Cases 1-6 are as follows:

(1) For Case 1, the numerical example with constant disturbance $\left(d_{\alpha 1}=5 \mathrm{deg} / \mathrm{s}^{2}, d_{\alpha 2}=10 \mathrm{deg} / \mathrm{s}^{2}, d_{\alpha 3}=\right.$ $\left.15 \mathrm{deg} / \mathrm{s}^{2}, \dot{d}_{\alpha i}=0, i=1,2,3\right)$ is simulated. The parameters of $u_{\alpha i}$ are chosen as $k_{\alpha i}^{P}=3.2, k_{\alpha i}^{D}=1.3, k_{\alpha i}^{I}=0(i=1,2,3)$, which corresponds to the situation without IC.

(2) Case 2 is the same as Case 1, except for $k_{\alpha i}^{I}=1(i=$ $1,2,3)$. That is, IC is applied for the numerical example with constant disturbance.

(3) Case 3 is the same as Case 2, except for time-varying $d_{\alpha i}$ that are given as follows:

$$
d_{\alpha i}=5 \sin (0.05 i \pi t)+5 i\left(\mathrm{deg} / \mathrm{s}^{2}\right), \quad i=1,2,3 .
$$

Note the three points: (a) as shown in (35), the three helicopters are subject to different disturbances; (b) initial disturbances $d_{\alpha i}(0)=5 i \mathrm{deg} / \mathrm{s}^{2}, i=1,2,3$; (c) to verify the results of Theorem 8 , the disturbances used in Case 2 satisfy conditions $\lim _{t \rightarrow+\infty} \dot{d}_{\alpha i}=0$ for each $i=1,2,3$, while those used in Case 3 do not.

(4) For Case 4, $u_{\alpha i}$ without IC are applied to the experiment platform shown in Figure 1. The parameters of $u_{\alpha i}$ are chosen as $k_{\alpha i}^{P}=5, k_{\alpha i}^{D}=8, k_{\alpha i}^{I}=0(i=1,2,3)$.

(5) Case 5 is the same as Case 4, except for $k_{\alpha i}^{I}=3(i=$ $1,2,3)$. That is, IC is applied to the experiment platform.

(6) For Case 6, in the experiments, the ADSs of helicopters 2 and 3 are activated and the same control laws as for Case 5 are applied.

It is worthwhile noting that ADS has a dramatic effect on the motion of helicopter body, whether it is static or moving. This point is easily seen from the mechanical structure of helicopter as shown in Figure 2 and is also verified by the following experiments.

4.1. Case 1: Numerical Simulation without Using IC. The numerical simulation results for this case are presented in Figure 5. As shown in subfigures (a) and (b) therein, because IC is not adopted in control design to attenuate constant disturbances, neither tracking error nor synchronization error converges to a small neighborhood of zero. More specifically,

(1) for the elevation axis, the magnitude of tracking error (of each helicopter) is greater than $5.5 \mathrm{deg}$ when $t \geq 20 \mathrm{sec}$ and is steady as $t$ increases. The synchronization error between any pair of the three helicopters is of a magnitude greater than 1 deg when $t \geq 20 \mathrm{sec}$ and is steady as $t$ increases. The reason is that the constant disturbance of the helicopter is different from each other and is not attenuated by IC.

(2) as shown in subfigures (c) and (d) of Figure 5, the voltage applied to either front motor or back motor, with a value equal to the maximum acceptable voltage $24 \mathrm{~V}$ at the beginning $(t \leq 0.8 \mathrm{sec})$, has a magnitude smaller than $24 \mathrm{~V}$ for all $t>0.8 \mathrm{sec}$ (smaller than $3 \mathrm{~V}$ for all $t>2 \mathrm{sec}$ ).

(3) as shown in subfigures (e) and (f), the attained accuracies of the FTC estimators are $\left|\ddot{\alpha}_{d}-\widehat{\ddot{\alpha}}_{d}\right| \leq$ $0.0074 \mathrm{deg} / \mathrm{s}^{2}$ and $\max _{1 \leq i \leq 3}\left|\dot{e}_{\alpha i}-\hat{\dot{e}}_{\alpha i}\right| \leq 0.003 \mathrm{deg} / \mathrm{s}$ that corresponds to Lemma 2 for FTC estimators (7) and (8) with finite time $t_{c}$ (in this case, $t_{c} \leq 7 \mathrm{sec}$ and $\max _{1 \leq i \leq 3} t_{c i} \leq 2.5 \mathrm{sec}$ for (7) and (8), resp.).

4.2. Case 2: Numerical Simulation with Using IC for Constant Disturbances. In this case, IC is adopted in control design to attenuate constant disturbances. The corresponding simulation results are presented in Figure 6, demonstrating that both tracking and synchronization performance are dramatically improved, compared with that achieved in Case 1. A detailed analysis of the results is given as follows:

(1) For the elevation axis, when $t \geq 20 \mathrm{sec}$, the magnitude of tracking error (of each helicopter) is smaller than $0.03 \mathrm{deg}$ and the synchronization error between any two helicopters has a magnitude smaller than $0.01 \mathrm{deg}$. Points (1) and (2) of Theorem 8 are verified by these results.

(2) The voltage applied to either front motor or back motor, with a value equal to the maximum acceptable voltage $24 \mathrm{~V}$ at the beginning $(t \leq 0.8 \mathrm{sec})$, is of a magnitude less than $3 \mathrm{~V}$ for all $t>2 \mathrm{sec}$.

(3) The result of FTC estimator (7) is the same as that in Case 1 and hence omitted. The attained accuracies of FTC estimator (8) are $\max _{1 \leq i \leq 3}\left|\dot{e}_{\alpha i}-\widehat{\dot{e}}_{\alpha i}\right| \leq 0.009 \mathrm{deg} / \mathrm{s}$ and the finite convergence time satisfies $\max _{1 \leq i \leq 3} t_{c i} \leq$ $15 \mathrm{sec}$.

4.3. Case 3: Numerical Simulation with Using IC for TimeVarying Disturbances. In this case, IC is adopted in control design to attenuate time-varying disturbances. The corresponding simulation results are presented in Figure 7. Although subject to the time-varying disturbances shown in (35), both tracking and synchronization performance are improved compared with that achieved in Case 1, where the disturbances are constants. However, compared with that achieved in Case 2, where the disturbances are constants and also attenuated by IC, both tracking and synchronization performance are degraded observably. A detailed analysis of the results is given as follows:

(1) For the elevation axis, when $t \geq 20 \mathrm{sec}$, the magnitude of tracking error (of each helicopter) is smaller than 1.0 deg; that is,

$$
\left|\widetilde{\alpha}_{i}(t)\right|<1.0 \mathrm{deg}, \quad \forall t>20 \mathrm{sec}, \quad i=1,2,3,
$$




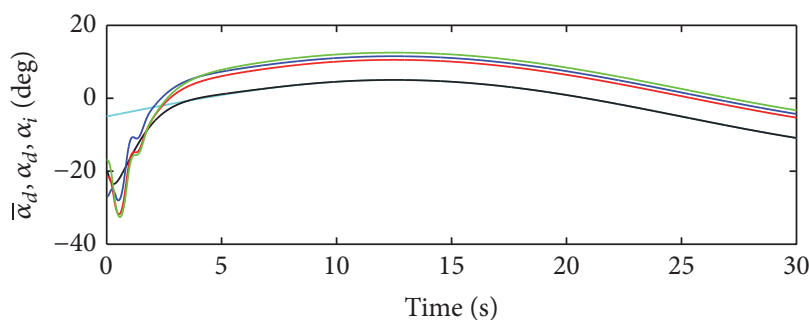

(a)

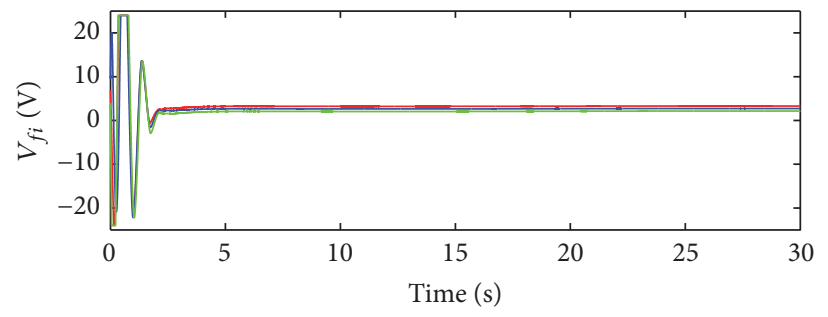

(c)

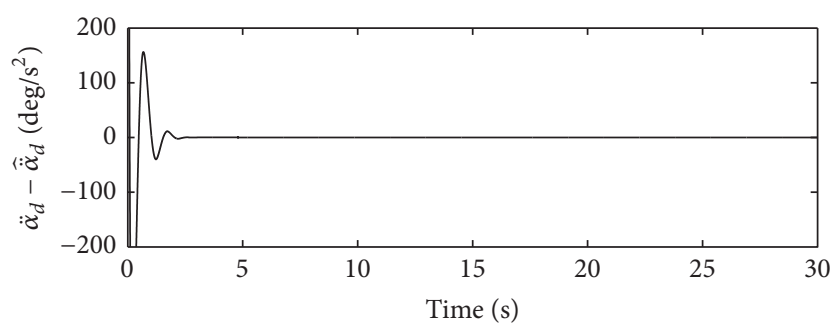

(e)

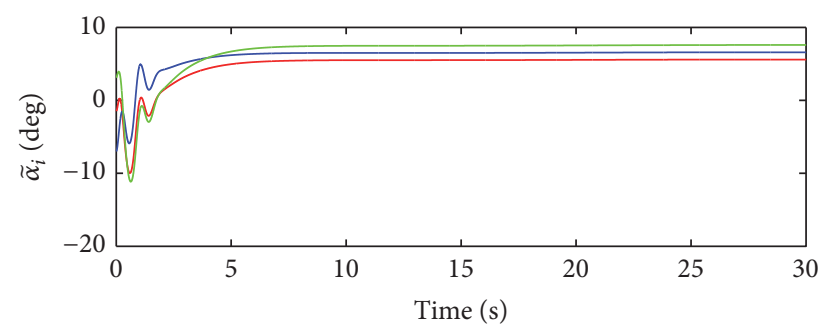

(b)

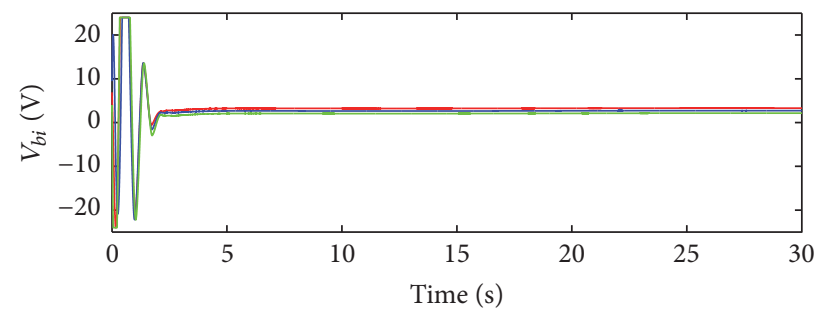

(d)

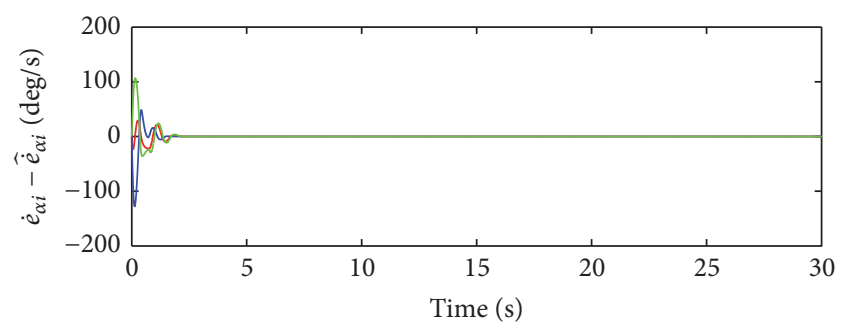

(f)

FIGURE 5: Numerical simulations results for Case 1 ( $\bar{\alpha}_{d}$-cyan, H0-black, H1-red, H2-blue, and H3-green): (a) elevation angular position trajectory, (b) elevation angle tracking error, (c) voltage of the front motor, (d) voltage of the back motor, (e) estimation error of the elevation angular acceleration of $\mathrm{H} 0$, and (f) estimation errors of the first-order derivative of LNSE of H1-H3.

and the synchronization error between any two helicopters has a magnitude smaller than $1.51 \mathrm{deg}$.

With parameters $k_{\alpha i}^{P}=3.2, k_{\alpha i}^{D}=1.3, k_{\alpha i}^{I}=1(i=$ $1,2,3)$ and (21), the following matrix $P_{\alpha i}$ can be obtained by resolving the Lyapunov equations (28):

$$
\begin{aligned}
P_{\alpha i} & =\left[\begin{array}{lll}
2.23 & 1.52 & 0.50 \\
1.52 & 3.10 & 0.63 \\
0.50 & 0.63 & 0.87
\end{array}\right], \\
\lambda_{\max }\left(P_{\alpha i}\right) & =4.43, \\
\lambda_{\min }\left(P_{\alpha i}\right) & =0.68 .
\end{aligned}
$$

With (4) and (35), we have $\bar{d}_{d \alpha}=0.041 \mathrm{deg} / \mathrm{s}^{3}$; then, along with (32) and (37), we get $b_{\alpha}$ in (27) that satisfies $b_{\alpha} \leq 6.41 \mathrm{deg}$ and the ultimate bound of the tracking errors in (26) satisfies $\|\widetilde{\boldsymbol{\alpha}}(t)\|_{2} \leq 9.16 \mathrm{deg}$. Then, points (1) and (3) of Theorem 8 are verified by (36).

(2) The voltage applied to either front motor or back motor, with a value equal to the maximum acceptable voltage $24 \mathrm{~V}$ at the beginning $(t \leq 0.8 \mathrm{sec})$, is of a magnitude less than $3.8 \mathrm{~V}$ for all $t>2 \mathrm{sec}$.

(3) The result of the FTC estimators is the same as that in Case 2 and hence omitted here.
4.4. Case 4: Experiment without Using IC. The experimental results for this case are presented in Figure 8. It is seen that the tracking error (of each helicopter) does not converge to a small neighborhood of zero and the synchronization performance is better than tracking performance. A detailed analysis of the results is given as follows:

(1) For the elevation axis, the magnitude of tracking error (of each helicopter) is greater than 3 deg for all $t>$ $10 \mathrm{sec}$; the synchronization error (between any two helicopters) is $\left|\alpha_{1}-\alpha_{2}\right| \leq 1.11 \mathrm{deg},\left|\alpha_{1}-\alpha_{3}\right| \leq$ $0.76 \mathrm{deg}$, and $\left|\alpha_{2}-\alpha_{3}\right| \leq 0.69 \mathrm{deg}$ for all $t>10 \mathrm{sec}$; the synchronization error between $\mathrm{H} 2$ and $\mathrm{H} 3$ has a smaller magnitude than that between $\mathrm{H} 1$ and $\mathrm{H} 2$ (or $\mathrm{H} 1$ and $\mathrm{H} 3$ ), which is an immediate consequence of the fact that $\mathrm{H} 2$ and $\mathrm{H} 3$ can obtain information from each other and both of them are equipped with ADSs, whereas $\mathrm{H} 1$ is not.

(2) The voltage applied to either the front or back motor, with a value equal to the maximum acceptable voltage $24 \mathrm{~V}$ at the beginning $(t \leq 6 \mathrm{sec})$, has a magnitude smaller than $21 \mathrm{~V}$ for all $t>6 \mathrm{sec}$.

4.5. Case 5: Experiment with Using IC. The experimental results for this case are presented in Figure 9. It is observed 


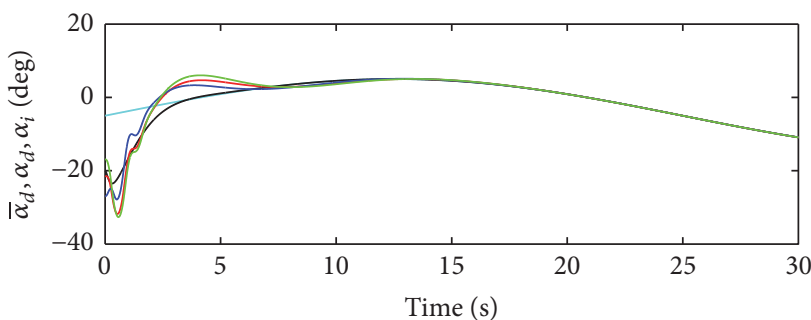

(a)

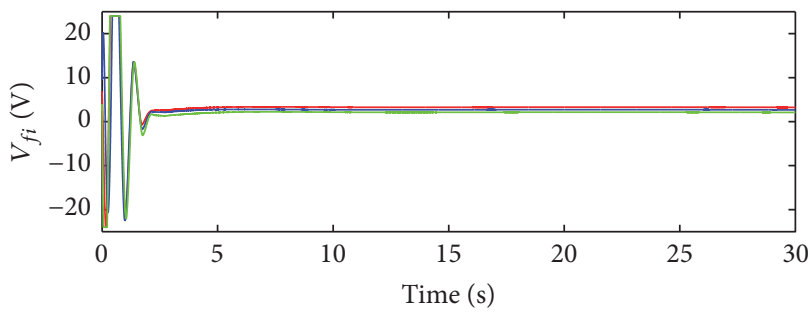

(c)

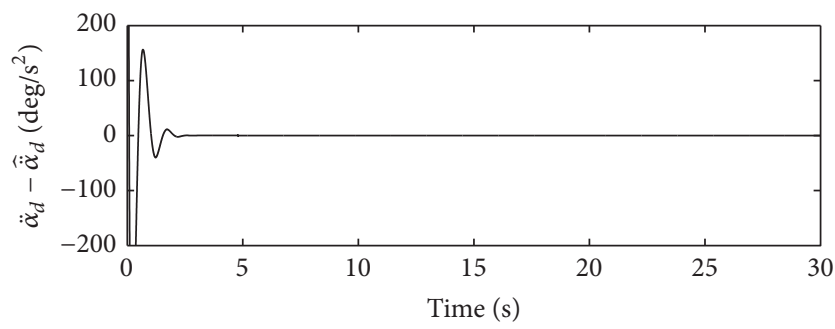

(e)

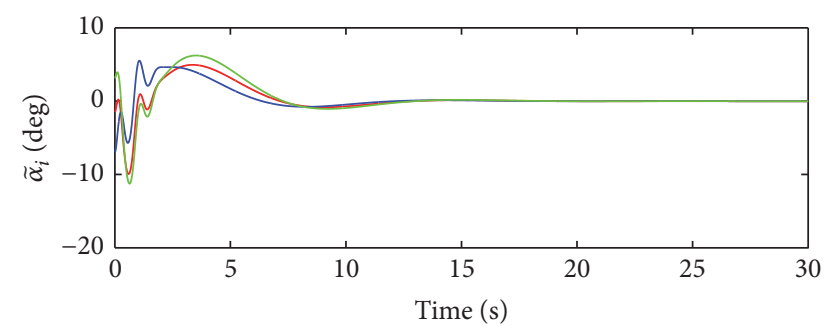

(b)

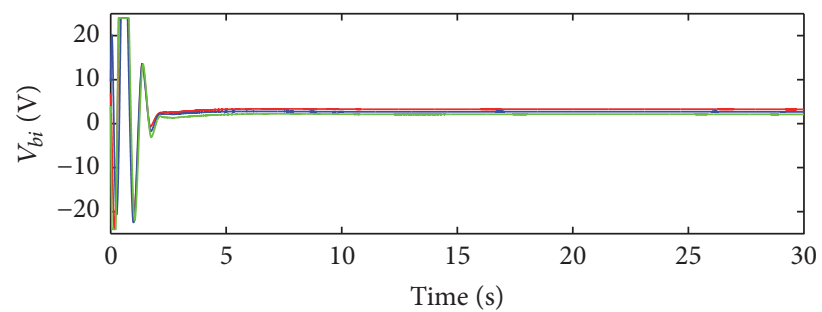

(d)

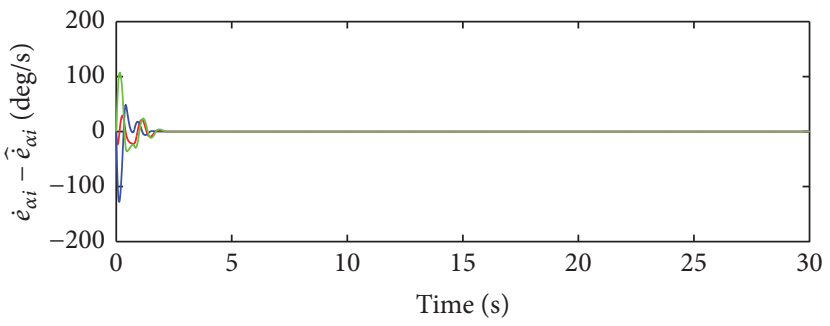

(f)

FIGURE 6: Numerical simulation results for Case 2 ( $\bar{\alpha}_{d}$-cyan, H0-black, H1-red, H2-blue, and H3-green): (a) elevation angular position trajectory, (b) elevation angle tracking error, (c) voltage of the front motor, (d) voltage of the back motor, (e) estimation error of the elevation angular acceleration of $\mathrm{H} 0$, and (f) estimation errors of the first-order derivative of LNSE of H1-H3.

that, compared with Case 4, both tracking and synchronization performance are improved because the disturbances are attenuated by IC in this case. More specifically,

(1) for the elevation axis, the magnitude of tracking error (of each helicopter) is smaller than $1.02 \mathrm{deg}$ for all $t>10 \mathrm{sec}\left(\widetilde{\alpha}_{1}=1.01 \mathrm{deg}, \widetilde{\alpha}_{2}=0.78 \mathrm{deg}\right.$, and $\widetilde{\alpha}_{3}=$ $0.66 \mathrm{deg}$ ); the synchronization error (between any two helicopters) is $\left|\alpha_{1}-\alpha_{2}\right| \leq 0.58 \mathrm{deg},\left|\alpha_{1}-\alpha_{3}\right| \leq 0.67 \mathrm{deg}$, and $\left|\alpha_{2}-\alpha_{3}\right| \leq 0.45 \mathrm{deg}$ for all $t>10 \mathrm{sec}$;

(2) the voltage applied to either the front or back motor, with a value equal to the maximum acceptable voltage $24 \mathrm{~V}$ at the beginning ( $t \leq 5 \mathrm{sec}$ ), has a magnitude smaller than $22 \mathrm{~V}$ for all $t>5 \mathrm{sec}$.

4.6. Case 6: With Activated ADS and the Same Controller as for Case 5. The ADSs equipped on helicopters 2 and 3 are activated in this case, whose dynamic positions along the arms of helicopters are shown in Figure 10, with the same initial position $-0.14 \mathrm{~m}(-0.14 \mathrm{~m}$ is the farthest possible position from propellers). This is different from the previous Case 4 and Case 5 where they are both fixed at $-0.14 \mathrm{~m}$. The experimental results for this case are presented in Figure 11. It is seen that motion of ADSs does not lead to dramatic performance degradation in the steady state of tracking and synchronization errors, compared with the performance achieved in Case 5. A detailed comparative analysis of the results is given as follows:

(1) For the elevation axis, the ultimate bound of either tracking error or synchronization error is nearly the same with that achieved in Case 5; the transient performance is degraded a little (this degradation is relatively obvious for helicopter 2 because the effect of the moving ADS on helicopter 4 is delivered to helicopter 2 which has no access to the desired trajectory as shown in Figure 4).

(2) The voltage applied to either the front or back motor, with a value equal to the maximum acceptable voltage $24 \mathrm{~V}$ at the beginning $(t \leq 6 \mathrm{sec}$ ), has a magnitude smaller than $23.5 \mathrm{~V}$ for all $t>5 \mathrm{sec}$.

To demonstrate the different steady-state tracking performance clearly, the maximum magnitudes of tracking errors that appeared in each case during time interval $t \in[20,30]$ are shown in Table 3 (Cases 1-3) and Table 4 (Cases 4-6). As shown in these tables, the tracking accuracy is improved in numerical simulations and experiments by IC. 


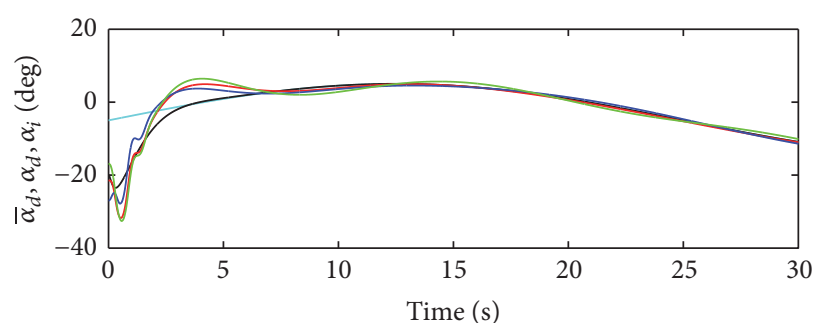

(a)

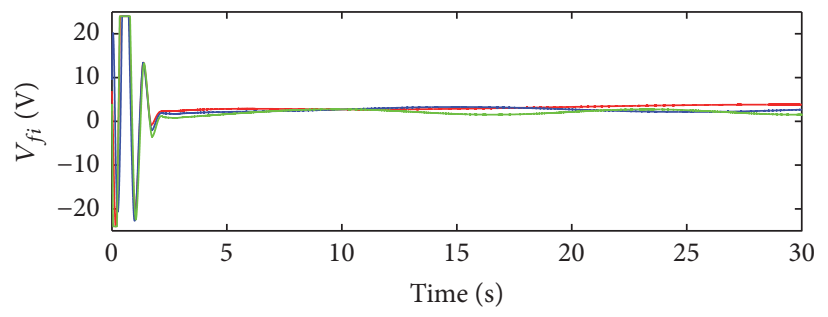

(c)

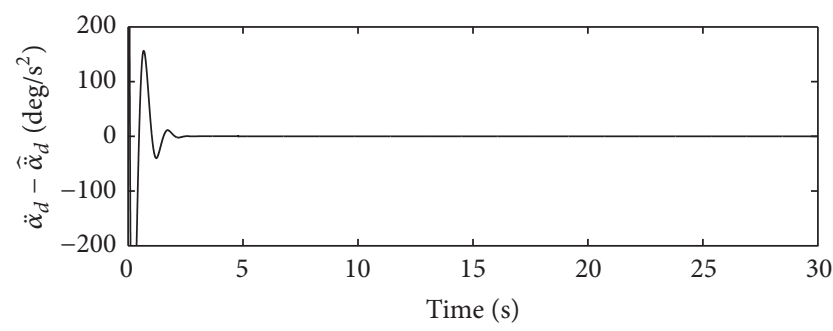

(e)

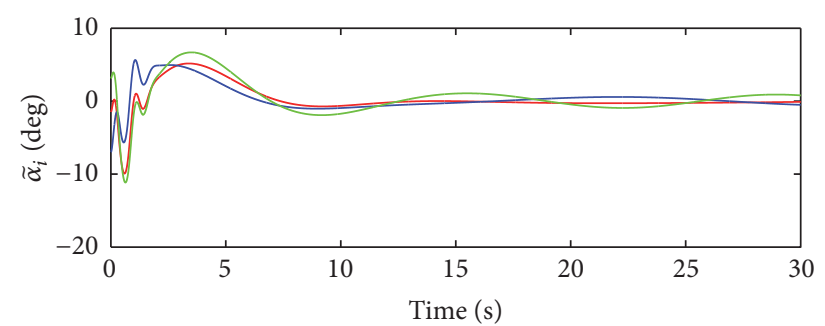

(b)

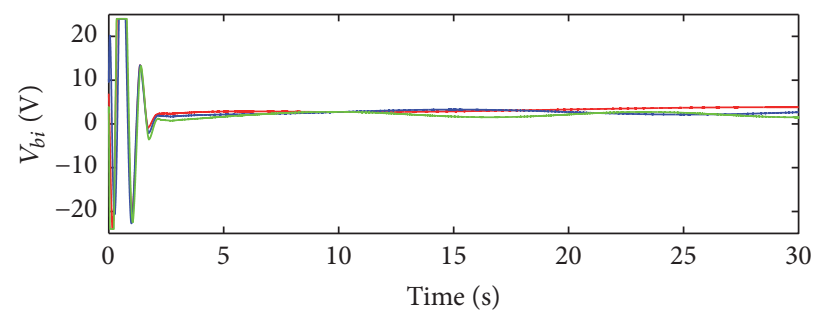

(d)

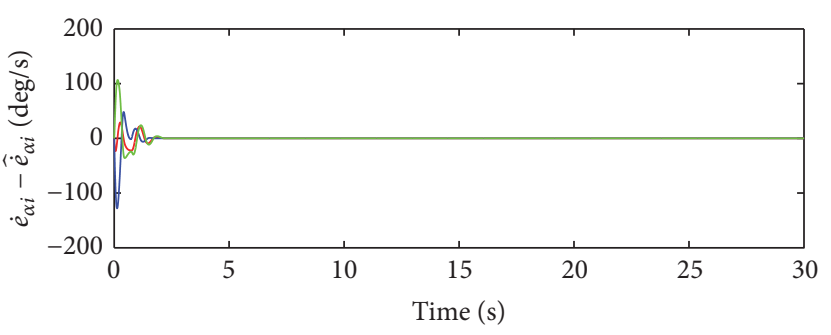

(f)

FIGURE 7: Numerical simulation results for Case 3 ( $\bar{\alpha}_{d}$-cyan, H0-black, H1-red, H2-blue, and H3-green): (a) elevation angular position trajectory, (b) elevation angle tracking error, (c) voltage of the front motor, (d) voltage of the back motor, (e) estimation error of the elevation angular acceleration of $\mathrm{H} 0$, and (f) estimation errors of the first-order derivative of LNSE of H1- $\mathrm{H} 3$.

TABLE 3: Maximum magnitudes of $\widetilde{\alpha}_{i}, i=1,2,3$, in simulations for $t>20 \mathrm{~s}$ (deg).

\begin{tabular}{lccc}
\hline Case & $\mathrm{H} 1$ & $\mathrm{H} 2$ & $\mathrm{H} 3$ \\
\hline 1 & 5.58 & 6.59 & 7.59 \\
2 & 0.027 & 0.027 & 0.027 \\
3 & 0.28 & 0.62 & 0.95 \\
\hline
\end{tabular}

TABLE 4: Maximum magnitudes of $\widetilde{\alpha}_{i}, i=1,2,3$, in experiments for $t>20 \mathrm{~s}(\mathrm{deg})$.

\begin{tabular}{lccc}
\hline Case & $\mathrm{H} 1$ & $\mathrm{H} 2$ & $\mathrm{H} 3$ \\
\hline 4 & 2.99 & 3.40 & 3.12 \\
5 & 1.0 & 0.78 & 0.66 \\
6 & 0.89 & 0.69 & 0.94 \\
\hline
\end{tabular}

\section{Concluding Remarks}

The robust distributed consensus tracking controllers with integral action for multiple 3-DOF experimental helicopters without velocity measurements have been studied under the condition that only the desired angular position measurements are accessible to a small subset of the helicopters. Motivated by the effectiveness of the tracking controller with integral action in disturbances rejection for the single vehicle, the distributed controllers have been proposed by combining FTC estimators with distributed integral controllers. With using the FTC estimators, great accuracy and finite-time convergence have been achieved for the estimation of the lacking information. Meanwhile, the distributed controllers with integral action stabilized the tracking errors and rejected the input disturbances. Through analysing the closed-loop stability, the conditions ensuring zero-error tracking and the ultimate bound of errors for the general cases with nonzero error have been derived. It has been verified through the results of numerical simulations and experiments on platform of "three 3-DOF helicopters" that the tracking and synchronization accuracy have been improved by the proposed controllers with proper parameters.

Future work will focus on the design and experimental verification of bounded distributed controller for the platform under the directed communication graph with timedelay. The control parameters in (6) also need to be optimized to get the minimized ultimate bound of tracking errors. 


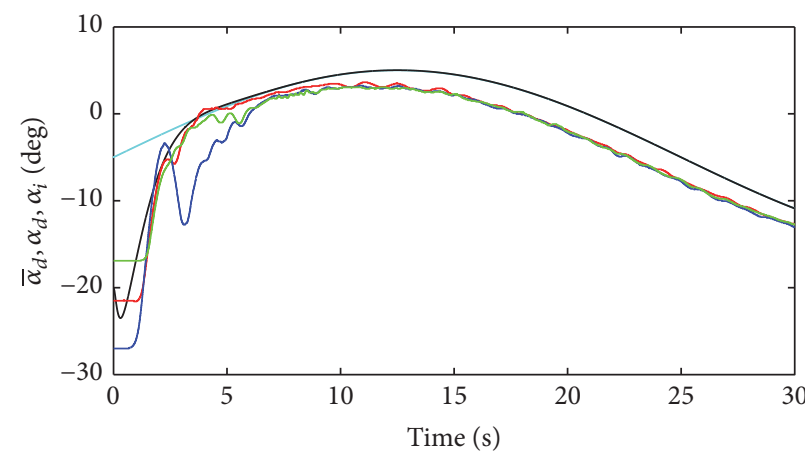

(a)

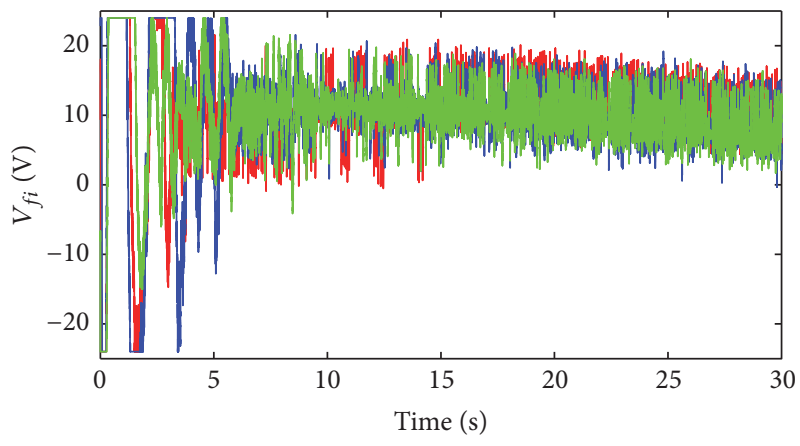

(c)

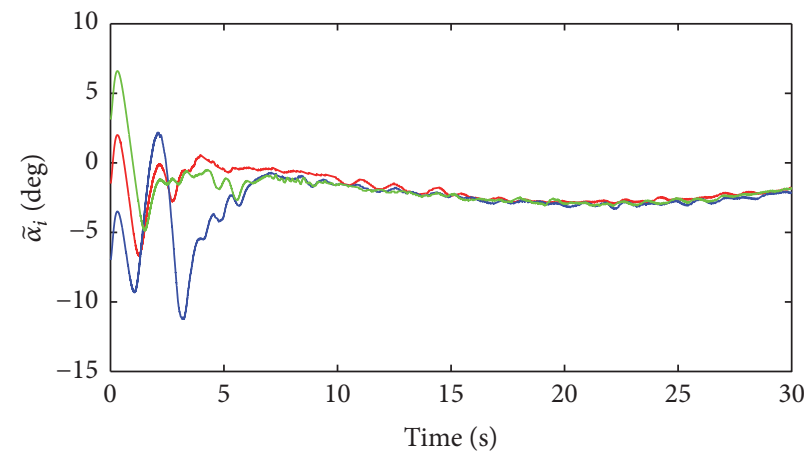

(b)

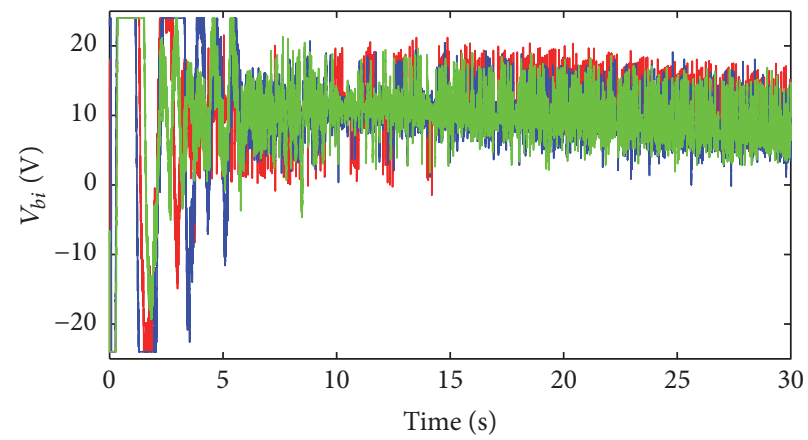

(d)

Figure 8: Experimental results for Case 4 ( $\bar{\alpha}_{d}$-cyan, H0-black, H1-red, H2-blue, and H3-green): (a) elevation angular position trajectory, (b) elevation angle tracking error, (c) voltage of the front motor, and (d) voltage of the back motor.

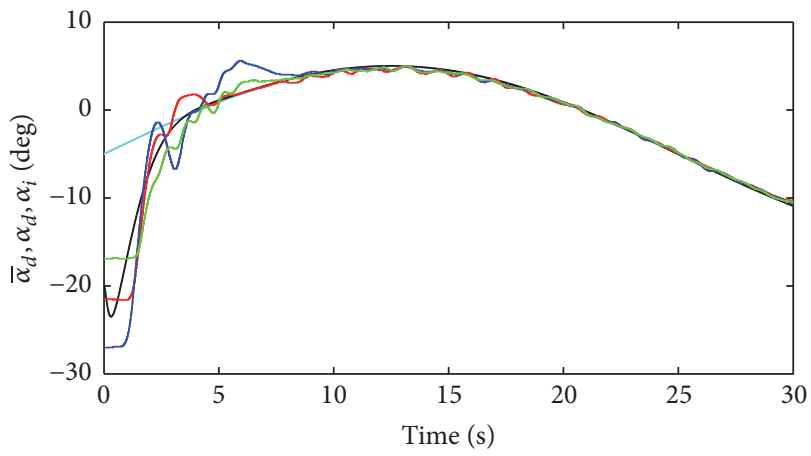

(a)

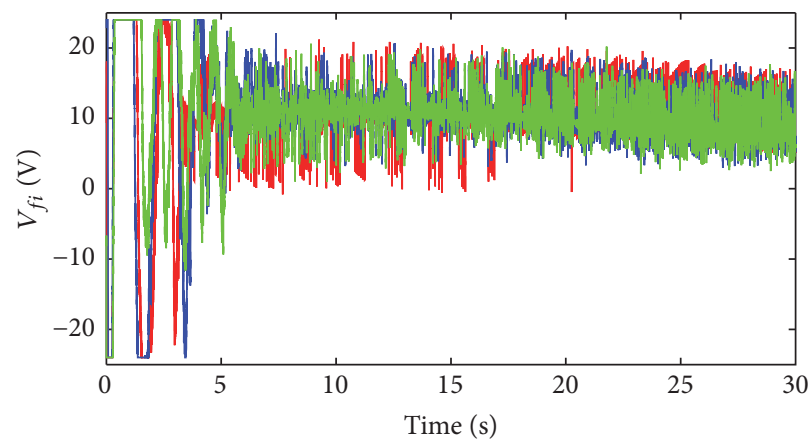

(c)

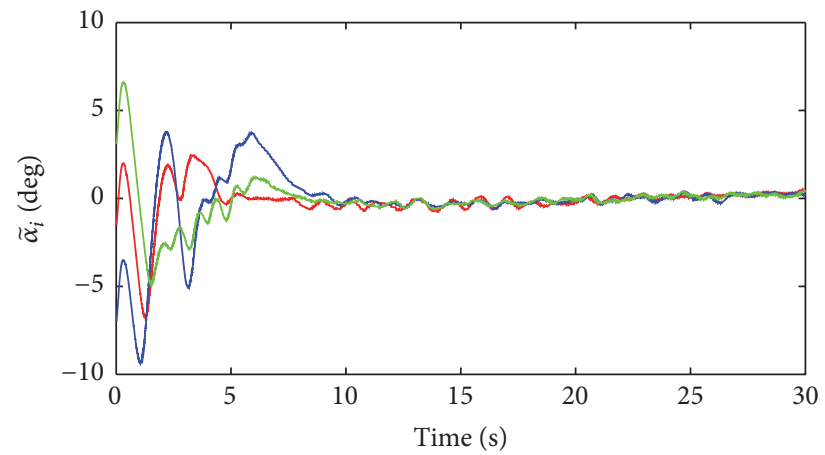

(b)

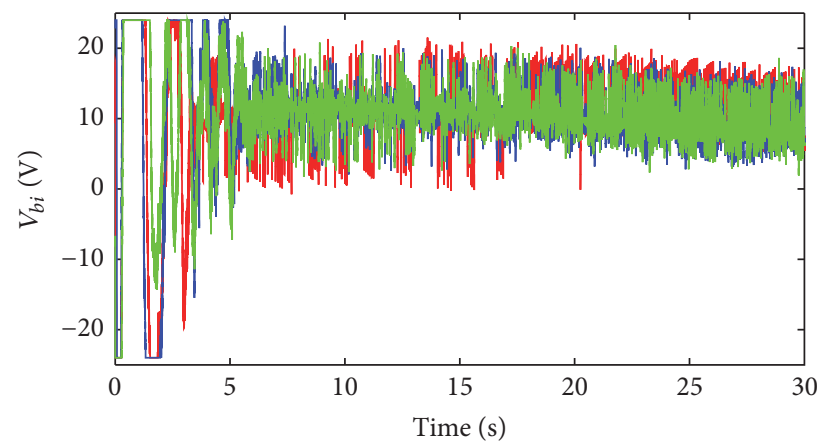

(d)

FIGURE 9: Experimental results for Case 5 ( $\bar{\alpha}_{d}$-cyan, H0-black, H1-red, H2-blue, and H3-green): (a) elevation angular position trajectory, (b) elevation angle tracking error, (c) voltage of the front motor, and (d) voltage of the back motor. 


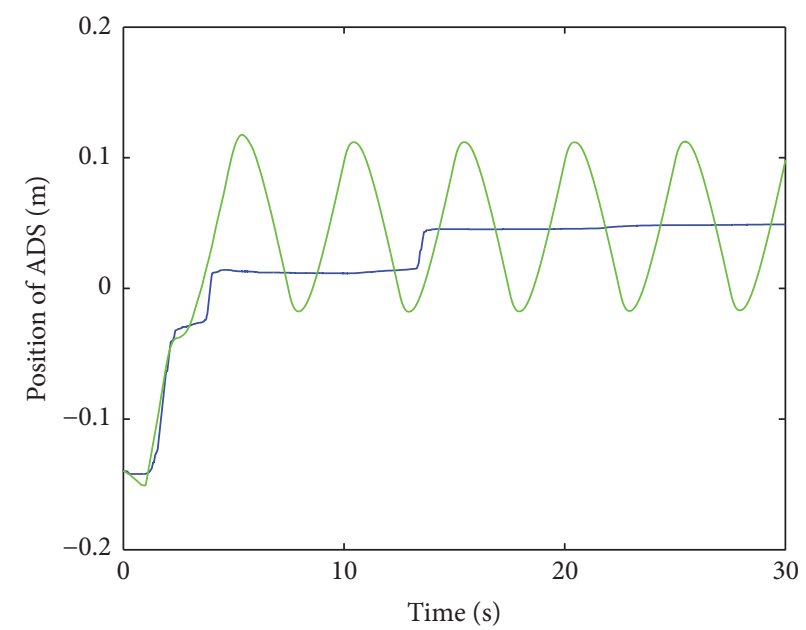

Figure 10: Positions of ADSs on helicopters 2 and 3: helicopter 2 (blue) and helicopter 3 (green).

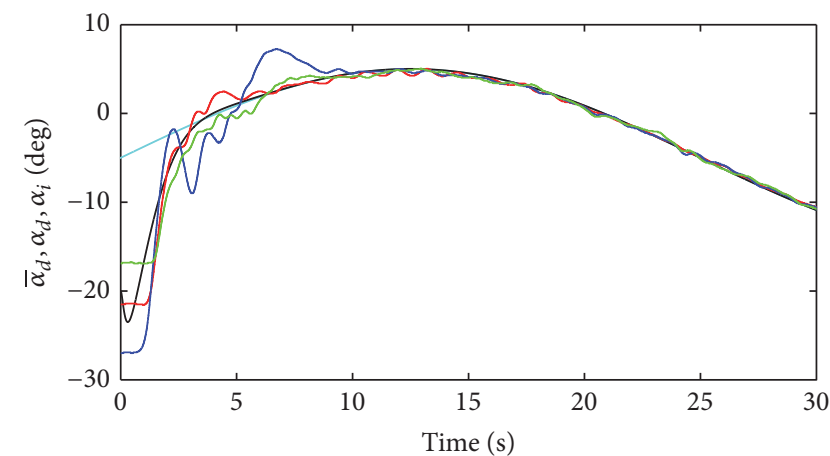

(a)

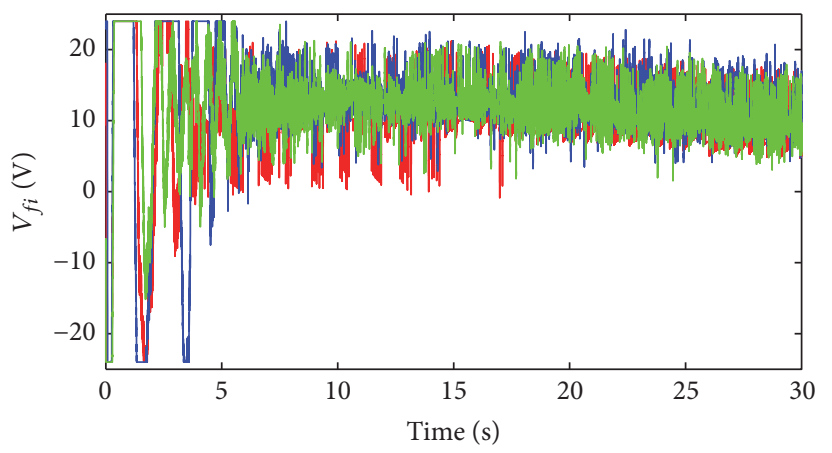

(c)

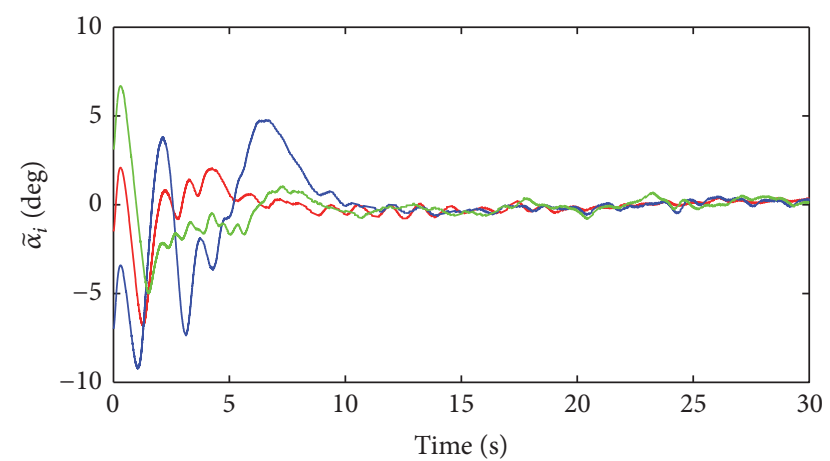

(b)

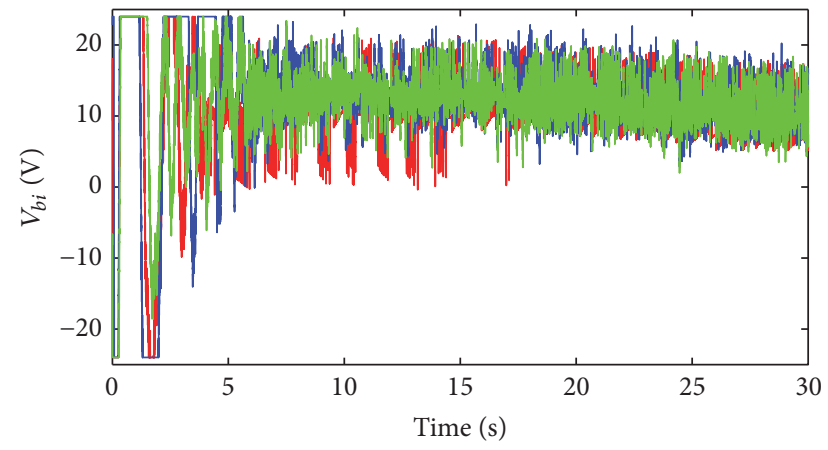

(d)

Figure 11: Experimental results for Case 6 ( $\bar{\alpha}_{d}$-cyan, H0-black, H1-red, H2-blue, and H3-green): (a) elevation angular position trajectory, (b) elevation angle tracking error, (c) voltage of the front motor, and (d) voltage of the back motor.

\section{Appendix}

A useful lemma used to show boundedness and ultimate bound of the solutions of some disturbed state equations is given as follows.
Lemma A.1 (see [5, Lemma 2]). Consider state solution $x(t)$ of the linear time-invariant equation:

$$
\dot{x}=A x+B u,
$$


where $x \in R^{m}$ is the state, $u \in R$ is the continuously differentiable input, and matrices $A \in R^{m \times m}$ and $B \in R^{m \times 1}$. If $A$ is Hurwitz, then

(1) system (A.1) is globally input-to-state stable (GISS); that is, if $u$ is bounded for all t, then $x(t)$ with any initial state $x\left(t_{0}\right)$ is also bounded for all $t$;

(2) there exist class $\mathscr{K} \mathscr{I}$ function $\gamma$ and time $T \geq 0$ (dependent on $x\left(t_{0}\right)$ and $\|u\|_{\mathscr{L}_{\infty}}=\sup _{t \geq 0}|u(t)|<\infty$ ) such that $x(t)$ with any $x\left(t_{0}\right)$ satisfies

$$
\begin{aligned}
\|x(t)\|_{2} & \leq \gamma\left(\|x(0)\|_{2}, t\right), \quad \forall t_{0} \leq t \leq t_{0}+T, \\
\|x(t)\|_{2} \leq \frac{2 \lambda_{\text {max }}(P)\|B\|_{2}\|u\|_{\mathscr{L}_{\infty}}}{\theta} \sqrt{\frac{\lambda_{\text {max }}(P)}{\lambda_{\text {min }}(P)}}, & \forall t \geq t_{0}+T,
\end{aligned}
$$

where $0<\theta<1, \lambda_{\max }(P)$, and $\lambda_{\text {min }}(P)$ are the maximum and minimum eigenvalues of the symmetric positive-definite matrix $P \in R^{m \times m}$ which is the solution of the Lyapunov equation:

$$
P A+A^{T} P=-I_{m} .
$$

\section{Notations}

$\alpha_{i}, \beta_{i}$ : The elevation angle and pitch angle, respectively (rad)

$\dot{\alpha}_{i}, \dot{\beta}_{i}: \quad$ The elevation angle rate and pitch angle rate, respectively ( $\mathrm{rad} / \mathrm{s})$

$J_{e i}, J_{p i}$ : The moments of inertia about elevation axis and pitch axis, respectively $\left(\mathrm{kg} \cdot \mathrm{m}^{2}\right)$

$K_{f i}: \quad$ The force constant of motor-propeller combination $(\mathrm{N} / \mathrm{V})$

$l_{a i}: \quad$ The distance from travel axis and elevation axis to the center of helicopter body $(\mathrm{m})$

$l_{h i}: \quad$ The distance from pitch axis to either motor $(\mathrm{m})$

$m_{i}$ : The effective mass of helicopter body $(\mathrm{kg})$

g: The gravitational acceleration constant, $\left(9.81 \mathrm{~m} / \mathrm{s}^{2}\right)$

$f_{\alpha i}, f_{\beta i}$ : The lumped uncertainties and disturbances (LUDs) acting on elevation and pitch channels, respectively $(\mathrm{N} \cdot \mathrm{m})$

$V_{f i}, V_{b i}$ : The voltages applied to the front motor and back motor, respectively; the voltage limit for the motors is $24 \mathrm{~V}$ (i.e., $\left.\left|V_{f i}\right| \leq 24 \mathrm{~V},\left|V_{b i}\right| \leq 24 \mathrm{~V}\right)(\mathrm{V})$

$V_{s i}, V_{d i}$ : The sum and the difference of $V_{f i}$ and $V_{b i}$, respectively $(\mathrm{V})$.

\section{Competing Interests}

The authors declare that they have no competing interests.

\section{Acknowledgments}

This work is supported by the National Natural Science Foundation of China under Grants nos. 61304016 and 61305132, the Fundamental Research Funds for the Central Universities under Grant no. ZYGX2016KYQD102. The authors would like to thank Professor Hugh. T. Liu at UTIAS for generously providing the 3-DOF helicopter platform at FSC lab to verify the controllers developed in this paper.

\section{References}

[1] S. Khoo, L. Xie, and Z. Man, "Robust finite-time consensus tracking algorithm for multirobot systems," IEEE/ASME Transactions on Mechatronics, vol. 14, no. 2, pp. 219-228, 2009.

[2] W. Zhang, Z. Wang, and Y. Guo, "Robust consensus for uncertain multi-agent systems on directed communication topologies," in Proceedings of the 49th IEEE Conference on Decision and Control (CDC '10), pp. 6317-6322, Atlanta, Ga, USA, December 2010.

[3] G. Hu, "Robust consensus tracking of a class of second-order multi-agent dynamic systems," Systems \& Control Letters, vol. 61, no. 1, pp. 134-142, 2012.

[4] C. Zhang, H. Wu, J. He, and C. Xu, "Consensus tracking for multi-motor system via observer based variable structure approach," Journal of the Franklin Institute. Engineering and Applied Mathematics, vol. 352, no. 8, pp. 3366-3377, 2015.

[5] B. Zhu, H. H.-T. Liu, and Z. Li, "Robust distributed attitude synchronization of multiple three-DOF experimental helicopters," Control Engineering Practice, vol. 36, pp. 87-99, 2015.

[6] B. Zhu, Z. Li, H. H.-T. Liu, and H. Gao, "Robust second-order tracking of multi-vehicle systems without velocity measurements on directed communication topologies," in Proceedings of the American Control Conference (ACC '14), pp. 5414-5419, IEEE, Portland, Ore, USA, June 2014.

[7] B. Zhu, C. Meng, and G. Hu, "Robust consensus tracking of double-integrator dynamics by bounded distributed control," International Journal of Robust and Nonlinear Control, vol. 26, no. 7, pp. 1489-1511, 2016.

[8] A. Das and F. L. Lewis, "Distributed adaptive control for synchronization of unknown nonlinear networked systems," Automatica, vol. 46, no. 12, pp. 2014-2021, 2010.

[9] Y. Su and J. Huang, "Cooperative output regulation with application to multi-agent consensus under switching network," IEEE Transactions on Systems, Man, and Cybernetics Part B: Cybernetics, vol. 42, no. 3, pp. 864-875, 2012.

[10] M. Andreasson, D. V. Dimarogonas, H. Sandberg, and K. H. Johansson, "Distributed control of networked dynamical systems: static feedback, integral action and consensus," IEEE Transactions on Automatic Control, vol. 59, no. 7, pp. 1750-1764, 2014.

[11] B. Zhu, "On overshoot and integrators," IET Control Theory \& Applications, vol. 8, no. 8, pp. 633-640, 2014.

[12] A. N. Gündeş and E. C. Wai, "Low order integral-action controller synthesis," Systems \& Control Letters, vol. 60, no. 11, pp. 885-891, 2011.

[13] A. Levant, "Higher-order sliding modes, differentiation and output-feedback control," International Journal of Control, vol. 76, no. 9-10, pp. 924-941, 2003.

[14] A. Levant, "Non-homogeneous finite-time-convergent differentiator," in Proceedings of the 48th IEEE Conference on Decision and Control, pp. 8399-8404, Shanghai, China, December 2009.

[15] J. Shan, H.-T. Liu, and S. Nowotny, "Synchronised trajectorytracking control of multiple 3-DOF experimental helicopters," 
IEE Proceedings-Control Theory and Applications, vol. 152, no. 6, pp. 683-692, 2005.

[16] 3DOF Helicopter System, Product Information, Quanser Inc., Markham, Canada, 2002, http://www.quanser.com.

[17] A. T. Kutay, A. J. Calise, M. Idan, and N. Hovakimyan, "Experimental results on adaptive output feedback control using a laboratory model helicopter," IEEE Transactions on Control Systems Technology, vol. 13, no. 2, pp. 196-202, 2005.

[18] T. Kiefer, K. Graichen, and A. Kugi, "Trajectory tracking of a 3DOF laboratory helicopter under input and state constraints," IEEE Transactions on Control Systems Technology, vol. 18, no. 4, pp. 944-952, 2010.

[19] H. Ríos, A. Rosales, A. Ferreira, and A. Dávila, "Robust regulation for a 3-DOF helicopter via sliding-modes control and observation techniques," in Proceedings of the American Control Conference (ACC '10), pp. 4427-4432, Baltimore, Md, USA, July 2010.

[20] A. F. de Loza, H. Ríos, and A. Rosales, "Robust regulation for a 3-DOF helicopter via sliding-mode observation and identification," Journal of the Franklin Institute, vol. 349, no. 2, pp. 700718, 2012.

[21] H. Liu, G. Lu, and Y. Zhong, "Robust LQR attitude control of a 3-DOF laboratory helicopter for aggressive maneuvers," IEEE Transactions on Industrial Electronics, vol. 60, no. 10, pp. 46274636, 2013.

[22] Z. Li, Z. Duan, G. Chen, and L. Huang, "Consensus of multiagent systems and synchronization of complex networks: a unified viewpoint," IEEE Transactions on Circuits and Systems I: Regular Papers, vol. 57, no. 1, pp. 213-224, 2010.

[23] W. Ren and Y. Cao, "Distributed coordination of multi-agent networks," in Communications and Control Engineering: Emergent Problems, Models, and Issues, Springer, Berlin, Germany, 2011.

[24] P. Pepe and I. Karafyllis, "Converse Lyapunov-Krasovskii theorems for systems described by neutral functional differential equations in Hale's form," International Journal of Control, vol. 86, no. 2, pp. 232-243, 2013.

[25] P. Wei, Q. Guan, W. Yu, and L. Wang, "Easily testable necessary and sufficient algebraic criteria for delay-independent stability of a class of neutral differential systems," Systems and Control Letters, vol. 57, no. 2, pp. 165-174, 2008.

[26] S. Liu, L. Xie, and F. L. Lewis, "Synchronization of multiagent systems with delayed control input information from neighbors," Automatica, vol. 47, no. 10, pp. 2152-2164, 2011. 


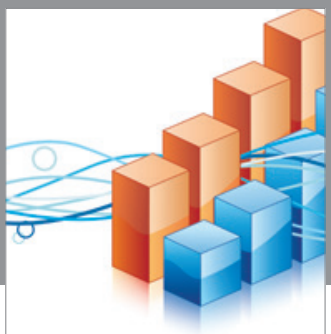

Advances in

Operations Research

vatem alat4

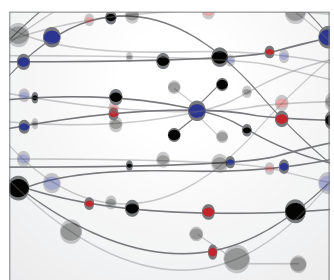

\section{The Scientific} World Journal
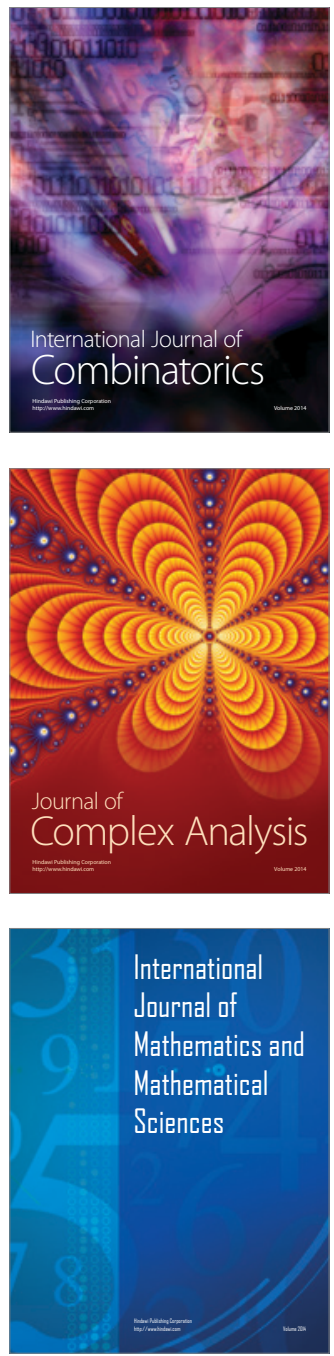
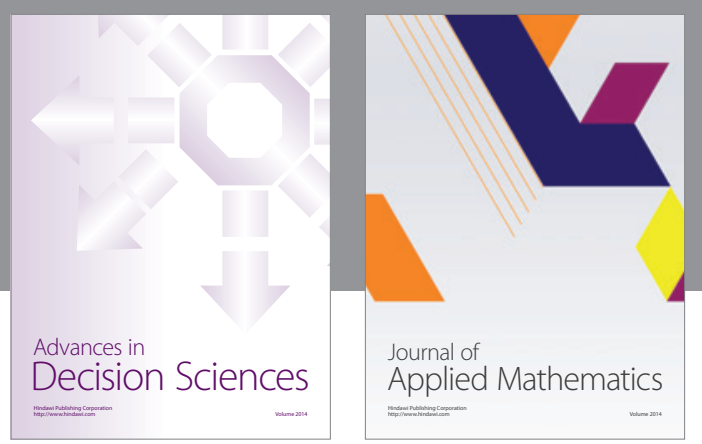

Algebra

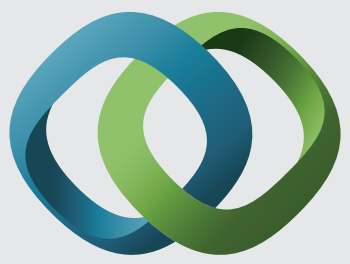

\section{Hindawi}

Submit your manuscripts at

http://www.hindawi.com
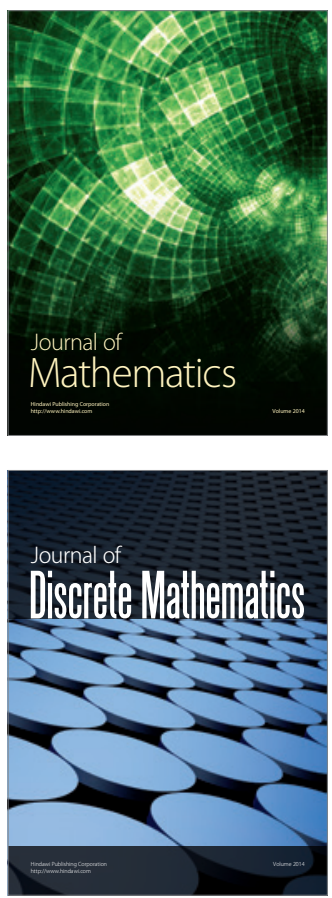

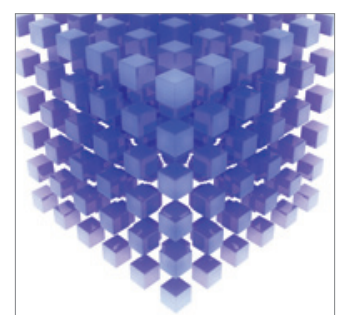

Mathematical Problems in Engineering
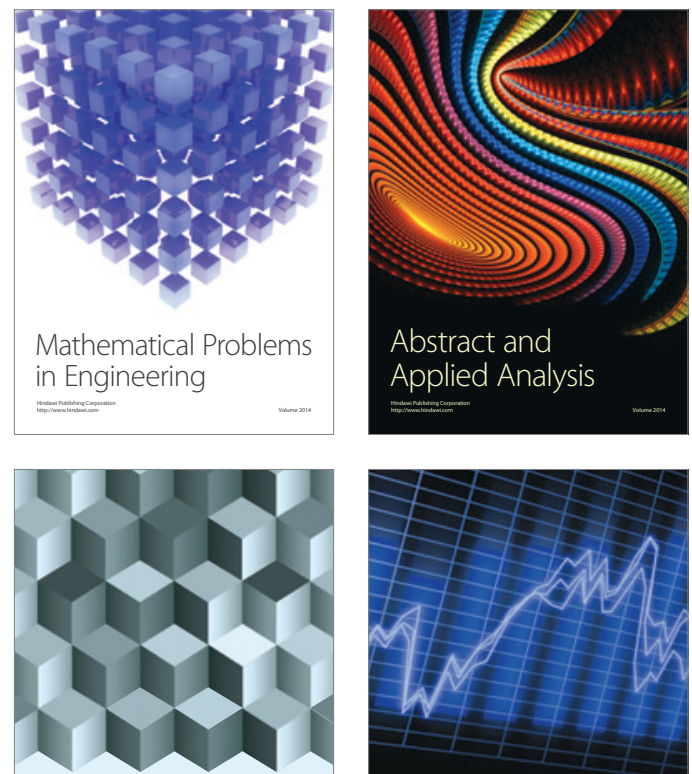

Journal of

Function Spaces

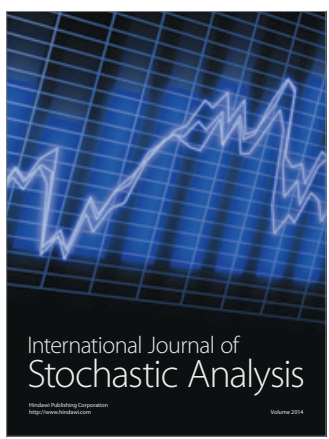

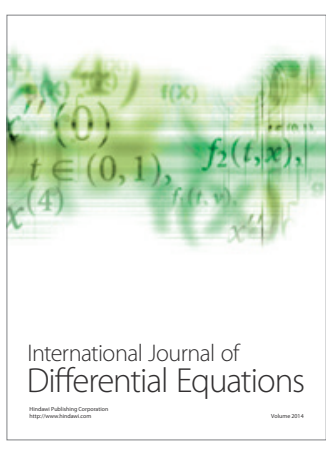
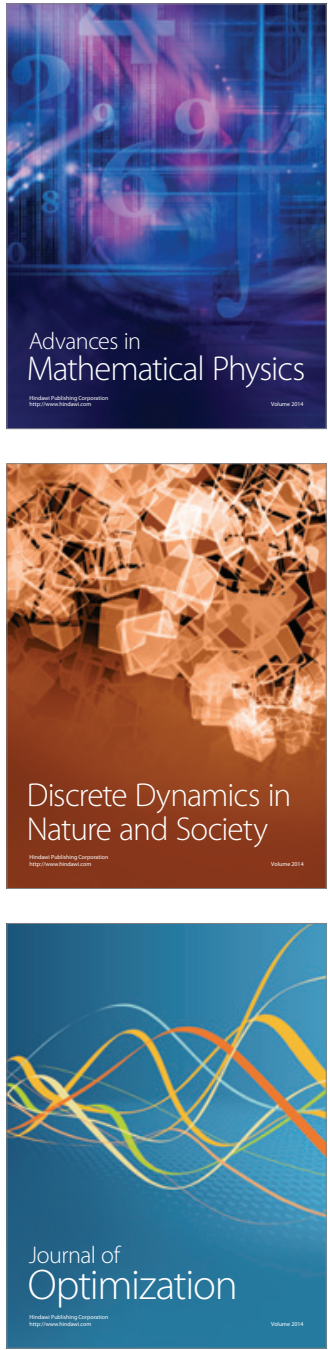